\title{
Soliton generation by internal tidal beams impinging on a pycnocline: laboratory experiments
}

\author{
Matthieu J. Mercier ${ }^{1,6} \dagger$, Manikandan Mathur ${ }^{2,3,1}$, Louis Gostiaux ${ }^{2}$, \\ Theo Gerkema ${ }^{4}$, Jorge M. Magalhães ${ }^{5}$, José C. B. Da Silva ${ }^{5}$ \\ and Thierry Dauxois ${ }^{6}$ \\ ${ }^{1}$ Department of Mechanical Engineering, Massachusetts Institute of Technology. 77 Massachusetts \\ Avenue. Cambridge, MA 02139. USA \\ ${ }^{2}$ Laboratoire des Ecoulements Geophysiques et Industriels (LEGI). UMR 5519 CNRS-UJF-INPG. \\ 21 rue des Martyrs, 38000 Grenoble. France \\ ${ }^{3}$ Laboratoire de Météorologie Dynamique, École Polytechnique, 91128 Palaiseau, France \\ ${ }^{4}$ Royal Netherlands Institute for Sea Research (NIOZ), PO Box 59, 1790 AB Den Burg, \\ Texel, The Netherlands \\ ${ }^{5}$ CIMAR/CIIMAR, Centro Interdisciplinar de Investigacão Marinha e Ambiental and Departamento de \\ Geociências, Ambiente e Ordenamento do Território, Universidade do Porto, Rua do Campo Alegre. \\ 687, 4169-007 Porto, Portugal \\ ${ }^{6}$ Laboratoire de Physique de l'École Normale Supérieure de Lyon. Université de Lyon, CNRS, \\ 46 Allée d'Italie. F-69364 Lyon CEDEX 07. France
}

(Received 4 August 2011; revised 2 February 2012; accepted 14 April 2012: first published online 29 June 2012)

In this paper, we present the first laboratory experiments that show the generation of internal solitary waves by the impingement of a quasi-two-dimensional internal wave beam on a pycnocline. These experiments were inspired by observations of internal solitary waves in the deep ocean from synthetic aperture radar (SAR) imagery, where this so-called mechanism of 'local generation' was argued to be at work, here in the form of internal tidal beams hitting the thermocline. Nonlinear processes involved here are found to be of two kinds. First, we observe the generation of a mean flow and higher harmonics at the location where the principal beam reflects from the surface and pycnocline; their characteristics are examined using particle image velocimetry (PIV) measurements. Second, we observe internal solitary waves that appear in the pycnocline, detected with ultrasonic probes; they are further characterized by a bulge in the frequency spectrum, distinct from the higher harmonics. Finally, the relevance of our results for understanding ocean observations is discussed.

Key words: internal waves, ocean processes, solitary waves

\section{Introduction}

Internal solitary waves (ISWs) are among the most noticeable kinds of internal waves in the ocean. Their surface manifestation may be visible even from spacecraft (see, for example, Jackson 2007). They often appear in groups, and the 
groups themselves usually appear regularly, every tidal period. This points to a tidal origin: barotropic tidal flow over topography creates internal tides, which, while propagating away from their source, may steepen and split up into ISWs. This is the common picture, in which the internal tide is regarded as a horizontally propagating, interfacial wave. In the early 1990s, New \& Pingree $(1990,1992)$ showed the first evidence of a very different generation mechanism of ISWs. The origin is still tidal, but the mechanism involves an internal tidal beam, generated over the continental slope, which first propagates downward, then reflects from the ocean floor, and finally, as its energy goes upward, impinges on the seasonal thermocline. Here it creates a depression, which, while propagating away, steepens and evolves into ISWs. This interpretation has been corroborated by synthetic aperture radar (SAR) imagery, showing a great increase of ISWs in the central Bay of Biscay, just beyond the area where the beam is expected to approach the surface (New \& Da Silva 2002; Da Silva, New \& Azevedo 2007). Recently, the same mechanism has been proposed to explain ISWs off Portugal (Da Silva et al. 2007) and in the Mozambique Channel (Da Silva, New \& Magalhaes 2009).

In this paper we present results on laboratory experiments - carried out at the LEGI Coriolis Platform in Grenoble in 2008 - that were set up to create ISWs by this very mechanism, i.e. by an internal wave beam impinging on a pycnocline. At the beginning of the generation process lies the internal 'tidal' beam, generated here with a recently designed wavemaker (Gostiaux et al. 2007; Mercier et al. 2010), which creates a well-defined monochromatic unidirectional beam. We varied the stratification, i.e. the strength, depth and thickness of the pycnocline which was set up above a constantly stratified lower layer. For each stratification we carried out experiments with different forcing frequencies, thus changing the angle the beam makes with the vertical. We note here that the wave beam generated by the wavemaker directly models the upward-propagating tidal beam that impinges on the ocean pycnocline.

In the set-up of the experiments, we were guided by theoretical studies of the subject. The process starts at the wavemaker, from which the internal-wave beam originates. So long as it propagates through the layer of uniform stratification, no significant changes are expected to occur, because a unidirectional wave beam propagating in a uniformly stratified Boussinesq fluid satisfies not only the linear but also the nonlinear equations of motion (Tabaei \& Akylas 2003). However, when wave beams propagating in multiple directions intersect, nonlinear effects such as generation of higher harmonics occur (Tabaei, Akylas \& Lamb 2005; Jiang \& Marcus 2009). This situation occurs when beams cross or reflect from boundaries.

In our set-up, and in the ocean too, the first deformation of the beam is expected to happen when it leaves the layer of near-constant stratification, i.e. when it encounters the pycnocline. This strong inhomogeneity of the medium causes the beam to reflect partially from and within the pycnocline, a linear process that 'scatters' the beam. As a result, some of the energy stays behind in the pycnocline. It has been shown in theoretical studies that this forms the basis from which ISWs may later evolve (Gerkema 2001; Akylas et al. 2007). At the same time, the partial reflections (that occur when a unidirectional wave beam propagates through a region of non-constant stratification) within the pycnocline, as well as the full reflection from the upper surface, create junctions of crossing beams, and hence form a source of nonlinear generation of higher harmonics. In our laboratory experiments, we thus focused on these two features: first, the evolution of the pycnocline after the impact of the beam, and second, higher harmonics. In a way, they are contrasting phenomena. ISWs in the pycnocline are the nonlinear result of a depression that steepens, the depression itself 
originating from an essentially linear process (internal reflections in the pycnocline). On the other hand, higher harmonics waves behave as linear waves but find their origin in a nonlinear process at the junction of the main beam and its reflected counterpart. This distinction in behaviour has consequences for the tools by which they may be analysed; in particular, the application of harmonic analysis is well suited to extracting the essentially linear higher harmonic beams from the measurements, but cannot expect to yield anything clear on the genuinely nonlinear ISWs. The same is true for wave spectra. We discuss this further in $\$ \S 3$ and 4.

The linear regime of the problem we address, an internal wave beam impinging a continuously stratified pycnocline in a finite-depth tank, was studied in Mathur \& Peacock (2009). Their experimental results on wave beam ducting, a scenario where the incident energy tends to remain trapped in the pycnocline even after multiple reflections within the pycnocline, were in excellent agreement with the viscous theory. This linear process, as discussed in Gerkema (2001) and Akylas et al. (2007), serves as a precursor to the formation of solitons.

Previous experimental and theoretical studies have identified the key parameters governing the response of the pycnocline to the incident beam. Delisi \& Orlanski (1975) derived a parameter $\beta$ which they interpreted as the square of the ratio of the phase speed for interfacial waves and the horizontal phase speed of the incident beam. This interpretation is open for some debate (see Thorpe 1998). The experimental results of Delisi \& Orlanski (1975) show that the response of the pycnocline is largest when $|\beta| \approx 1$. In a different setting, in which the interfacial waves were assumed to be long rather than short, a similar criterion was identified in terms of a parameter $\gamma$, defined as the ratio of the phase speed of interfacial waves and that of the first mode of the uniformly stratified lower layer (Gerkema 2001). We amplify on this below. Finally, Akylas et al. (2007), in a setting closer to that of Delisi \& Orlanski (1975) - their lower uniformly stratified layer being infinitely deep - recovered a parameter similar to $\beta$, which they call $\alpha$. In either setting, the parameter $g^{\prime}$ plays a key role, and we study its influence by examining different shapes and strengths of the pycnocline.

Recently, in a configuration similar to the present set-up, Grisouard, Staquet \& Gerkema (2011) modelled the local generation of ISWs with a nonlinear, nonhydrostatic numerical code (MIT-gem) and showed that higher mode ISWs (namely modes 2 and 3) may also be generated by this mechanism. Initially, the idea was to carry out numerical runs concurrently with the laboratory experiments, and the basic setting for the numerical model was chosen to be the same as in the laboratory. But, as shown in the rest of this paper, we observed some important qualitative differences (between the experiments and the simulations) that rendered a direct comparison impossible.

The present paper is organized as follows. In $\S 2$, a description of the experimental set-up and the measurement techniques is given. An analysis of the results from particle image velocimetry (PIV) is given in $\S 3$, where we focus on the mean flow and harmonics. Results from the ultrasonic probes are presented in $\S 4$; they provide a quantitative view of the pycnocline displacements and the ISWs. Finally, we end with a discussion of our results and conclusions in $\S 5$.

\section{Description of the experimental set-up}

The experiments were carried out at the Coriolis Platform of LEGI, Grenoble (see figure 1 for a schematic of the experimental set-up). This facility is a $13 \mathrm{~m}$ diameter rotating basin that can be filled with salty water; during the process of 
(a)

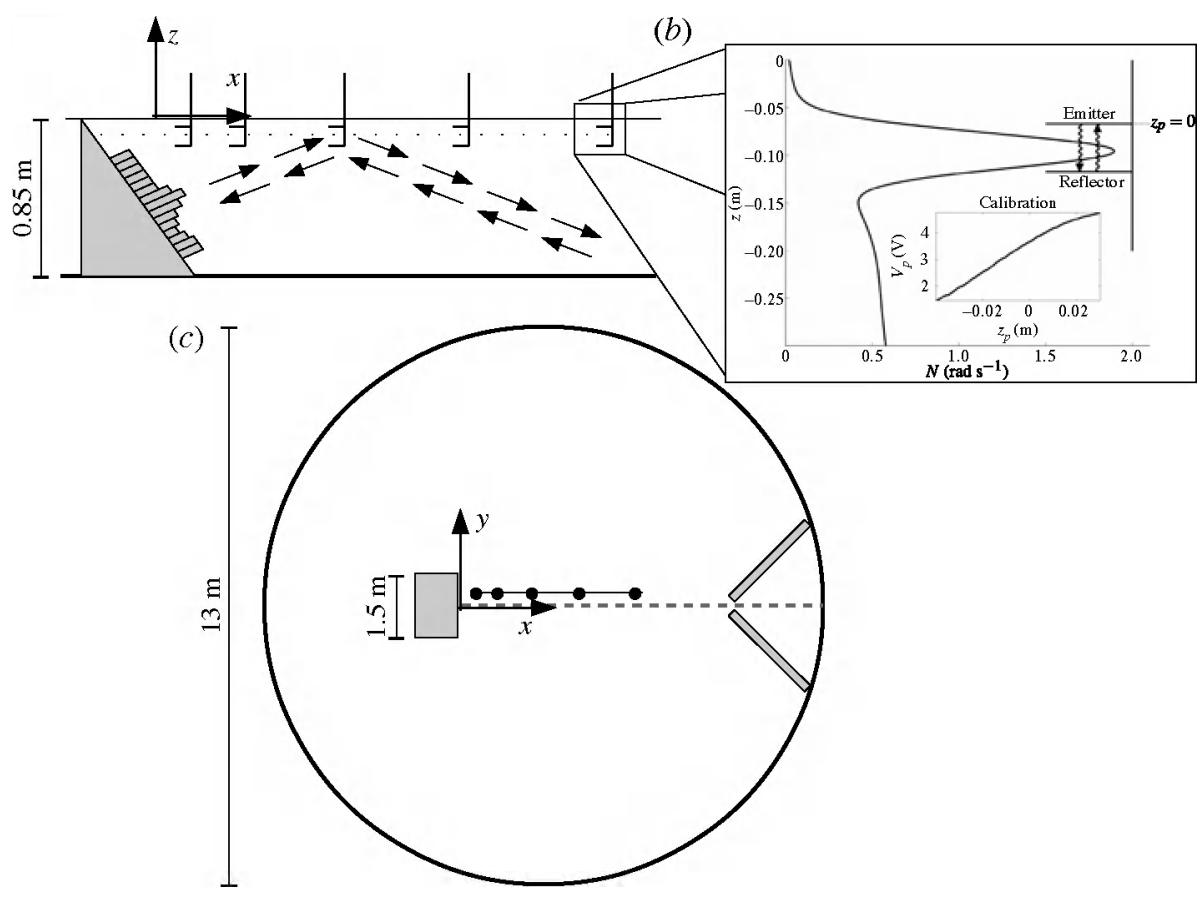

FIGURE 1. Schematic of the experiment: $(a)$ side view, $(b)$ close-up of the ultrasonic probe and its relative positioning with respect to the stratification, and $(c)$ top view. A typical calibration curve obtained by traversing the probe in quiescent medium is shown in the inset in $(b)$.

filling, the salinity can be changed, so that a vertically stratified layer is formed. The size of the experimental tank allowed visualization of the wave propagation over a large horizontal distance. Furthermore, the platform can serve as a turntable, but the experiments discussed in this paper were done without rotation.

\subsection{Experimental parameters}

A vertically stratified layer of height $80 \mathrm{~cm}$ was created by filling the tank from below with water whose salinity was gradually increased; this resulted in a layer whose density $\rho$ decreased from $1040 \mathrm{~kg} \mathrm{~m}^{-3}$ at the bottom to $1010 \mathrm{~kg} \mathrm{~m}^{-3}$ at the top. Thus, a layer of linearly stratified fluid was formed with a constant value $N_{0}=0.58 \mathrm{rad} \mathrm{s}^{-1}$ of the Brunt-Väisälä frequency,

$$
N=\sqrt{-\frac{g}{\rho_{0}} \frac{\mathrm{d} \rho}{\mathrm{d} z}},
$$

also referred to as the buoyancy frequency later on. The first experiments were carried out with this layer alone. It must be noted that the buoyancy frequency in this layer remained constant in subsequent days, except for the lowest part of the tank where it became slightly weaker with time, as can be seen in figure 10(a), due to molecular diffusion.

Then, a thin layer $(4 \mathrm{~cm})$ of fresh water $\left(\rho \approx 1000 \mathrm{~kg} \mathrm{~m}^{-3}\right)$ was added from above on top of the uniformly stratified layer. This served as the upper mixed layer, while 


\begin{tabular}{lccccccccccc}
\hline $\begin{array}{l}\text { EXP } \\
\text { no. }\end{array}$ & $\begin{array}{c}N_{\max } \\
\left(\mathrm{(ad} \mathrm{s}^{-1}\right)\end{array}$ & $\begin{array}{c}T \\
(\mathrm{~s})\end{array}$ & $\begin{array}{c}U_{\max } \\
\left(\mathrm{cm} \mathrm{s}^{-1}\right)\end{array}$ & $\begin{array}{c}k_{\eta} \\
\left(\mathrm{rad} \mathrm{m}^{-1}\right)\end{array}$ & $\begin{array}{c}\lambda_{x} \\
(\mathrm{~cm})\end{array}$ & $\begin{array}{c}C_{x} \\
\left(\mathrm{~cm} \mathrm{~s}^{-1}\right)\end{array}$ & $\gamma$ & $\omega / N_{0}$ & $\omega / N_{\max }$ & $R e$ & $A_{k}$ \\
03 & 0.58 & 21.6 & 0.74 & 31.7 & 39.5 & 1.83 & 0 & 0.50 & 0.50 & 232 & 0.80 \\
08 & 2.27 & 21.6 & 0.74 & 31.7 & 39.5 & 1.83 & 0.11 & 0.50 & 0.13 & 232 & 0.80 \\
16 & 1.52 & 21.6 & 0.74 & 31.7 & 39.5 & 1.83 & 0.19 & 0.50 & 0.19 & 232 & 0.80 \\
19 & 1.94 & 21.6 & 0.74 & 31.7 & 39.5 & 1.83 & 0.24 & 0.50 & 0.15 & 232 & 0.80 \\
21 & 1.83 & 41.8 & 0.40 & 32.8 & 73.8 & 1.77 & 0.17 & 0.26 & 0.08 & 122 & 0.87 \\
22 & 1.83 & 21.6 & 0.74 & 31.7 & 39.5 & 1.83 & 0.17 & 0.50 & 0.16 & 232 & 0.80 \\
25 & 1.83 & 16.9 & 0.68 & 32.2 & 30.4 & 1.80 & 0.17 & 0.64 & 0.20 & 217 & 0.60
\end{tabular}

TABLE 1. The name/number of the experiment; the maximum buoyancy frequency in the pycnocline $N_{\max }$; the forcing period $T$; the maximum velocity in the direction of propagation of the initial beam $U_{\max }$; the dominant wavenumber of the initial beam $k_{n}$; its horizontal wavelength, $\lambda_{x}=2 \pi / k_{\eta} \sin \theta$, where $\theta$ is the angle of the beam with the horizontal; and finally, the corresponding horizontal phase speed $C_{x}=\lambda_{x} / T$. Relevant nondimensional parameters are also provided: $\gamma$, a measure of the strength of the pycnocline (as explained in $\$ 4.2$ ); the ratio of forcing and stratification frequencies; the Reynolds number $R e=U_{m a x} / \nu k_{\eta}$; and the excursion parameter $A_{k}=U_{\max } k_{\eta} / \omega$.

the sharp transition of density between the two layers ensured the presence of a pycnocline. After a day, the pycnocline tended to lose its sharpness, and so new fresh layers were added after experiments, or sometimes the upper part of the water column was removed before adding fresh water. In this way, a whole range of different pycnoclines (in terms of peak value $N_{\max }$, thickness, and depth) was created, serving as varying background conditions during which the experiments were carried out. Examples of stratification profiles are provided in figure $10(a)$, and characteristic values are presented in table 1. Density measurements done after each experiment showed no significant changes compared to the profile taken before the experiment.

The ratios between $\omega$ and the stratification frequencies, $N_{0}$ and $N_{\max }$, are both of the order of $10^{-1}$ in our experiments, which is larger than those in the ocean. Typical values for the ocean are $N_{0}=10^{-3} \mathrm{rad} \mathrm{s}^{-1}$ and $N_{\max }=10^{-2} \mathrm{rad} \mathrm{s}^{-1}$, which give for the semi-diurnal tide $\left(\omega=1.4 \times 10^{-4} \mathrm{rad} \mathrm{s}^{-1}\right)$ ratios of the order of $10^{-1}$ and $10^{-2}$ respectively. This implies differences in the steepness of the incident internal wave beam, and the experimental results might not be directly applicable to the ocean.

\subsection{Incident internal wave beam}

In the basin, an internal wave beam directed upwards was generated by a wavemaker similar to that developed by Gostiaux et al. (2007), to which we refer for a detailed description. As described in Mercier et al. (2010), the internal-wave beam profile thus obtained is very similar to the solution derived by Thomas \& Stevenson (1972). Their profile has previously been shown to describe wave beams in the ocean after they propagate far from the continental shelf (Gostiaux \& Dauxois 2007). In our specific set-up, we can directly reproduce such a 'tidal' beam without topography and without barotropic flow.

Briefly, the device consists of 24 stacked PVC sheets of $2 \mathrm{~cm}$ thickness, shifted with respect to each other so as to form a wave pattern similar to the Thomas-Stevenson wave beam described in $\$ 4$ in Mercier et al. (2010). The PVC sheets in the wavemaker are connected via two eccentric camshafts, which, when subject to rotation, results in downward (phase) propagation of the wave pattern. At both ends of the stacking, there are sheets with zero amplitude of oscillation so that when measured 
along the wavemaker, the width of the generated beam is of the order of $33 \mathrm{~cm}$. The amplitude of oscillation of the plate in the centre of the beam is $3.5 \mathrm{~cm}$. Placed in a stratified fluid at an angle of $30^{\circ}$ with respect to the vertical, as depicted in figure $1(a)$, this wave motion is imparted to the fluid, thus producing a unidirectional internal wave beam at a single frequency. The device had a finite width of $1.5 \mathrm{~m}$ along the $y$-direction, resulting in three-dimensional effects close to the edges.

Quantitative measurements of the velocity field and the pycnocline displacements were made using PIV and ultrasonic probes, respectively. From the PIV velocity measurements described below, several properties of the beam can be estimated. We measured the maximum amplitude of the velocity $U_{\max }$ in the direction of propagation of the beam, and the dominant wavenumber $k_{\eta}$ associated with the profile. These properties, along with other relevant parameters in the experiments, are listed in table 1. It is interesting to notice that although our experiments are at quite low values for the Reynolds number, nonlinearity is expected to play an important part since the excursion parameter, $A_{k}=U_{\max } k_{\eta} / \omega$, which compares the fluid particle displacement with the characteristic wavelength of the problem, is close to 1 .

\subsection{Particle image velocimetry (PIV)}

Velocity fields have been obtained through PIV measurements done in the $(x O z)$ plane as shown in figure $1(c)$ (grey dashed line). Two CCD 12-bit cameras of resolution $1024 \times 1024$ record at $3 \mathrm{~Hz}$ images of particles illuminated by a $6 \mathrm{~W}$ continuous laser. Although the spatial resolution obtained is good, smaller than $1 \mathrm{~mm} \mathrm{pixel}{ }^{-1}$, it is only possible to visualize the linearly stratified region due to the strong optical distortions caused by the presence of the pycnocline.

Furthermore, the seeding of particles in the homogeneous surface layer is insufficient since the particles used here rather match the density range of the stratified layer. Some information can be extracted from floating particles at the free surface, as we will see in $\$ 3.1$, which allow us to extrapolate the velocity field from the the top of the linearly stratified region up to the free surface.

Experimental data are processed using open source CIVx algorithms by Fincham \& Delerce (2000) and the free Matlab toolbox UVMAT developed at LEGI. Some overlap between the fields of view of the two cameras allows for a horizontal merging of the velocity fields, leading to visualization windows of $1.6 \mathrm{~m} \times 0.8 \mathrm{~m}$.

\subsection{Ultrasonic probes}

The ultrasonic probes designed at LEGI (Michallet \& Barthélemy 1997) consisted of a sound emitter, which also acts as a receiver, and a reflector that were $0.05 \mathrm{~m}$ apart. Ten such probes were positioned at $x=0.18,0.39,0.62,0.89,1.20,1.254,1.59,2.09$, 2.80 and $4.0 \mathrm{~m}$ from the wave generator, respectively (see figure $1 a$ ). Each probe was mounted vertically on a plane that was slightly off-centre so as to not interfere with the PIV measurements, at a height that ensured that the emitter was in the mixed layer and the reflector in the lower constant stratification layer. The voltage output from the probe is proportional to the time taken for the sound to traverse its path from the emitter to the reflector and back to the emitter/receiver. This traversal time is directly proportional to the relative distance between the emitter and the pycnocline, if the pycnocline is infinitesimally sharp. In realistic scenarios, like the stratification in our experiments, where the pycnocline is of finite thickness, the output from the ultrasonic probe is a measure of the average (over $z$ ) displacement of the pycnocline at the specific $x$ location of the probe. 

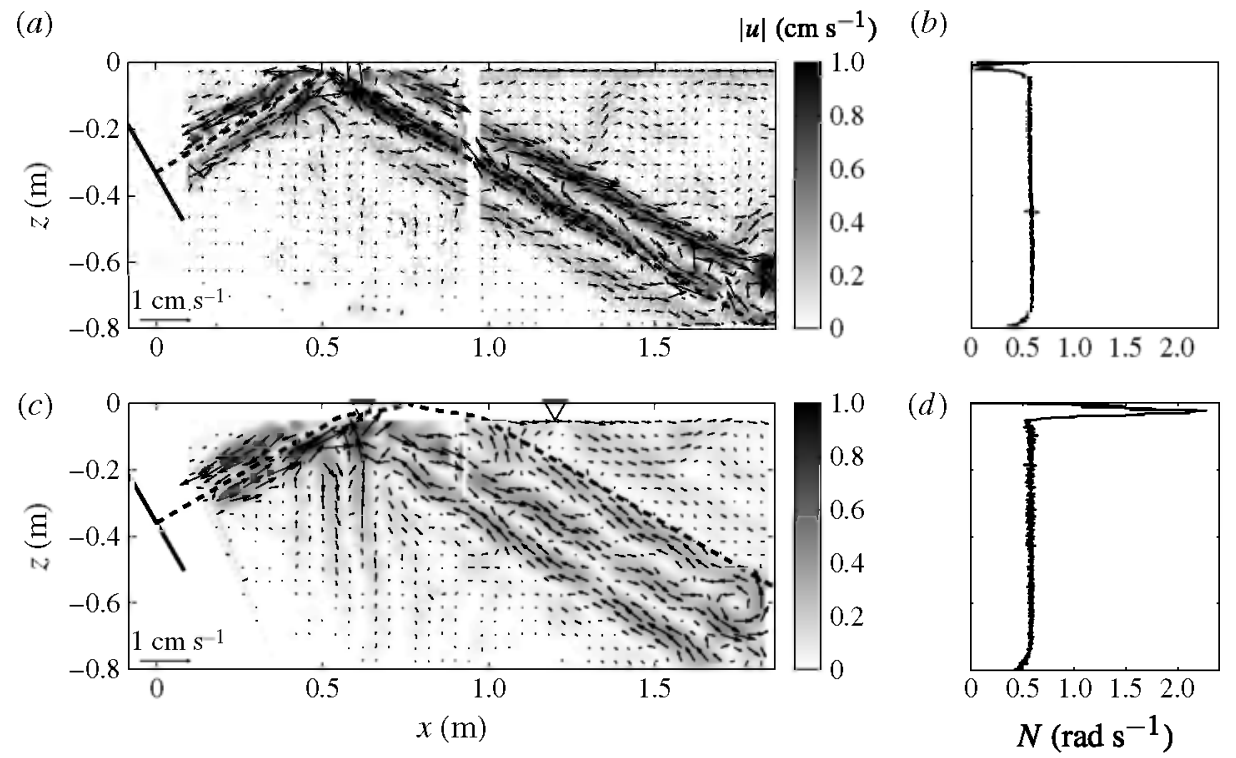

FIGURE 2. Comparison between experiments without ( $a, b, \mathrm{EXP03})$ and with a pycnocline $(c, d$, EXP08). The stratification profiles are shown in $(b)$ and $(d)$, and the corresponding measured velocity vectors and magnitude at $t=240 \mathrm{~s}$ after the start-up of the forcing in $(a)$ and $(c)$, respectively. The dashed lines represent the theoretical direction of internal-wave energy propagation, starting from the centre of the wavemaker, which is indicated by the solid line on the left. Insufficient overlap between the two cameras, around $x=0.9 \mathrm{~m}$, leads to a gap in the velocity field in $(a)$. The two inverted triangles in $(c)$ indicate the positions of the ultrasonic probes at $x=0.62 \mathrm{~m}$ and $x=1.20 \mathrm{~m}$.

To calibrate each ultrasonic probe, it is moved using a linear traversal mechanism up and down by known distances in a quiescent setting where there is no flow, and hence no pycnocline displacements. The output voltage is continuously recorded as a function of the position of the probe. This allows us to get a voltage $V_{p}$ versus relative position of the pycnocline $z_{p}$ relation for the probe (see figure $1 b$ ), and the procedure is done for every probe. Converting the output voltage from experiments to equivalent pycnocline displacements involves interpolation on the respective calibration curves.

It is important to note that the values thus found are a measure of the mean vertical displacements between the emitter and receiver. A mode trapped in the pycnocline will have maximum vertical excursions at a certain depth, while the excursions decline rapidly above and below. The acoustic probes, then, will not yield this maximum excursion but a lower value, because it also registers over parts in which the amplitude is declining. This reduction will affect the signal associated with even modes (i.e. modes 2, 4 etc.), in particular.

\section{Mean flow and harmonics}

Using PIV, we visualize what happens when the incident wave beam encounters the pycnocline and subsequently reflects from it. To demonstrate the significance of the presence of a pycnocline, we first present a qualitative comparison between settings without and with a pycnocline: see figure 2 . The effect of the pycnocline, as seen in the unfiltered PIV data presented in figure $2(c, d)$, is twofold. First, it shifts the beam 


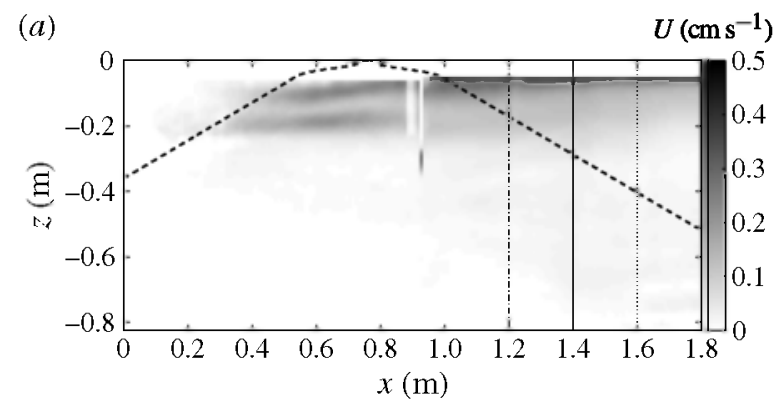

(b)

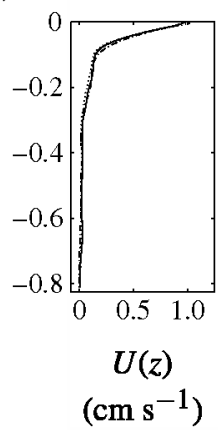

(c)

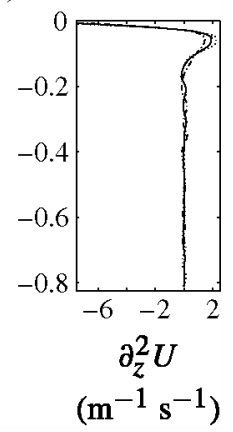

FIgURE 3. EXP08. (a) Mean horizontal velocity $U$ and vertical profiles of (b) $U$ and (c) $\partial^{2} U / \partial z^{2}$ extracted at $x=1.2 \mathrm{~m}$ (dash-dotted line), $1.4 \mathrm{~m}$ (continuous line) and $1.6 \mathrm{~m}$ (dotted line) from the wavemaker. The thick dashed line represents the theoretical direction of internal-wave energy propagation, starting from the centre of the wavemaker. Insufficient overlap between the two cameras, around $x=0.9 \mathrm{~m}$, leads to a gap in the velocity field.

slightly to the right because the beam propagates at a smaller angle with respect to the horizontal in the layer of strong stratification (as indicated by the dashed line which represents the theoretical path of energy propagation). Second, the reflected beam is broadened, and therefore less intense, when it emerges from the pycnocline. This is caused by (multiple) internal reflections: the beam reflects not only from the upper free surface but also from and within the pycnocline itself (Gerkema 2001; Mathur \& Peacock 2009).

As mentioned in the Introduction, two kinds of nonlinear phenomena are expected to take place during reflection at the pycnocline: the nonlinear evolution of the pycnocline displacements excited by the incoming beam, and the generation of higher harmonics at the junction between the incident and reflected beams. The latter occurs even if no pycnocline is present, namely upon reflection from the surface. As noted earlier, the uppermost part of the water column produces no visible signal with PIV in the experiments with a pycnocline. Therefore, we resort to the acoustic probes for visualizing and analysing the response at the pycnocline, the subject of $\$ 4$. In this section, we focus on the features extracted from the PIV data, notably the principal beam and its harmonics.

The harmonics cannot be properly interpreted unless we take into account another phenomenon that we found in our experiments: the occurrence of a mean flow in the upper layer. We therefore proceed to discuss this phenomenon first.

\subsection{Mean flow}

Apart from extracting the higher harmonics from the PIV data, harmonic analysis also reveals the presence of a mean Eulerian flow. The mean flow for EXP08, in which the cameras were placed close to the wavemaker, is shown in figure 3 . It is found to originate from the area of reflection of the forced beam at the pycnocline, and to spread further in the direction of wave propagation, but it remains restricted to the upper layer. The horizontal mean velocity displayed here is obtained by averaging over 12 forcing periods, starting at $t=360 \mathrm{~s}$ after the start-up of the forcing.

A complete description of the mean-flow field is not directly available as the PIV data lacks information in the upper few centimetres, typically $5 \mathrm{~cm}$, with the exact 


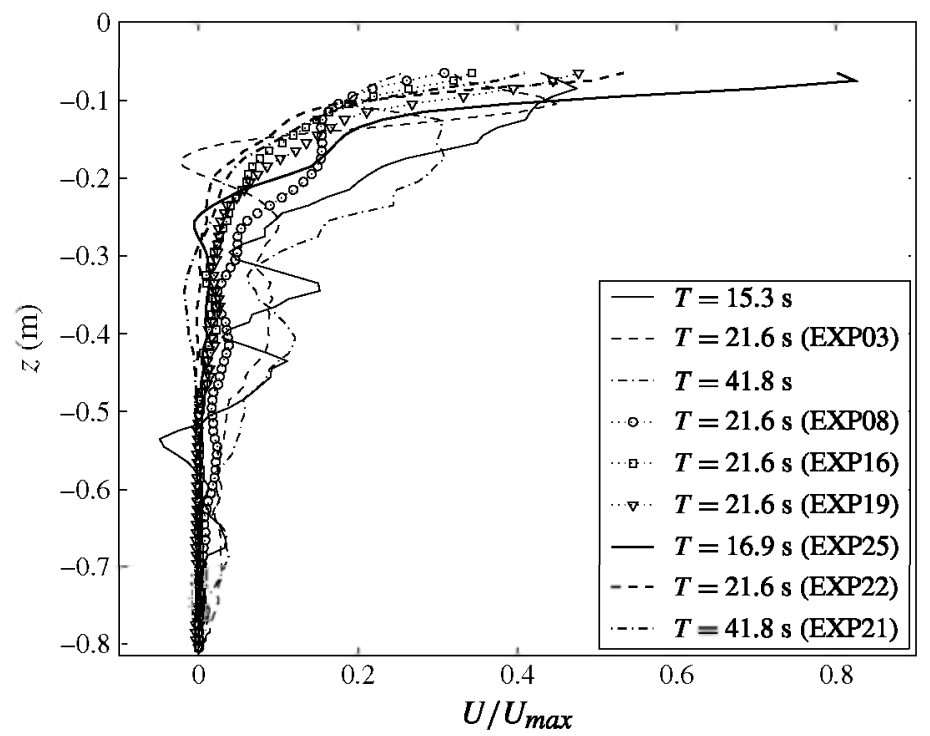

FIGURE 4. Vertical profiles of the mean horizontal velocity $U(z)$ extracted at $0.4 \mathrm{~m}$ away from the point of the impingement of the incident beam on the free surface, for various experiments. The two profiles (thin lines) not associated with any experiments are with the same pycnocline strength as EXP03.

value depending on the experiment. This is partially remedied by determining, from the raw PIV images, the strength of the mean flow at the free surface $(z=0)$ by following the particles trapped there by capillary effects. This allows us to establish the profile of the mean flow over the entire vertical, without having to extrapolate; we only interpolate (cubically) over the interval where data is lacking. Furthermore, we also impose the constraint of no shear at the free surface during interpolation. Figures $3(a)$ and $3(b)$ illustrate the resulting profiles. The second vertical derivative of the mean horizontal velocity, plotted in figure $3(\mathrm{c})$, is an important quantity as it features in (3.1), determining the vertical modal structure. A noticeable characteristic of the mean flow is that its amplitude varies with $x$, being maximum where it originates (at the area of reflection of the forced beam at the pycnocline) and then decaying slightly as you go away from the wavemaker. This evolution must be a signature of a three-dimensional structure of the flow; the amplitudes of the wave field at the forcing and higher harmonic frequencies, however, did not exhibit a similar decay in $x$. Hence, for the present purposes we assume the flow to be quasi-twodimensional.

Interestingly, the mean flow is present in all our experiments, including those without a pycnocline. In figure 4, we plot the vertical profile of the mean flow extracted at $0.4 \mathrm{~m}$ away from the point of impingement of the incident beam on the free surface for all experiments presented in table 1, and two extra for different frequencies in the cases with $\gamma=0$. The mean flow maximum velocity is of the same order as the maximum velocity of the incoming wave beam, but no clear effect of the varying pycnocline strength can be observed. So, it is the reflection from the surface, rather than the passage through the pycnocline, which lies at the origin of the mean flow. Furthermore, we verified that the mean flow is not present at the beginning of 
the experiment and is noticeable only after the beam impinges on the pycnocline and surface.

In none of the experiments is an appreciable mean flow found near the bottom of the tank, even though there, too, beam reflection takes place. However, since PIV measurements are not possible in the bottom $5 \mathrm{~mm}$ of the fluid, we cannot determine the flow in the vicinity of the wave-induced bottom boundary layer of thickness $\sqrt{v / \omega}$, of the order of $2 \mathrm{~mm}$ in our case. One could argue that nonlinear effects are stronger at the pycnocline due to the strengthening of the wave beam in regions of stronger stratification, and this may result in a net deposit of momentum. In figure $2(a, c)$, the first reflected beam coming from the pycnocline/free surface, when arriving at the tank bottom, is not much weaker than the incident forced beam. Hence, the contrast between the presence of a mean flow near the surface and its absence near the bottom is presumably due to the different nature of the boundaries, a free surface versus a solid boundary (i.e. free-slip versus no-slip). These explanations, however, must remain hypotheses since our experiments do not allow us to draw any firm conclusions. As a matter of fact, mean flow generation near a solid boundary has already been observed in experiments and numerical simulations of barotropic flow over a threedimensional topography (King, Zhang \& Swinney 2009). Nevertheless, the mechanism at play in our case is different since we do not have any topography, there is no barotropic forcing, and the mean flow generated is in the same plane as the incoming wave beam.

Other experiments dedicated to this mean flow are needed to better understand its cause and its possibly three-dimensional structure, as well as its dependence on various parameters (impinging beam angle/profile, strength of the pycnocline etc.) As discussed in the following sections, incorporating the effects of the mean flow plays a crucial role in the interpretation of the higher harmonics and other nonlinear waves in our experiments.

\subsection{Higher harmonics}

Here, we present a harmonic analysis of the PIV data from three experiments that were carried out on the same day, hence with nearly identical density profiles (all corresponding to $\gamma=0.17$ ), but at different forcing frequencies: $\omega_{f}=0.15 \mathrm{rad} \mathrm{s}^{-1}$ (EXP21), $\omega_{f}=0.29 \mathrm{rad} \mathrm{s}^{-1} \quad(\mathrm{EXP} 22), \omega_{f}=0.37 \mathrm{rad} \mathrm{s}^{-1}$ (EXP25). These three experiments had the cameras placed further away from the wavemaker than in EXP08, and hence provide a more complete view of the region after reflection from the pycnocline.

In EXP25 $\left(\omega_{f} / N_{0}=0.64\right)$, presented in figure 5, the principal beam at the forcing frequency reflects from the pycnocline and surface, and broadens due to internal reflections. The dominant wavenumber in the reflected beam, $k_{x}^{(r)} \simeq 10.7 \mathrm{rad} \mathrm{m}^{-1}$, is much smaller than the corresponding value in the incident wave beam, $k_{x}^{(i)}=$ $20.7 \mathrm{rad} \mathrm{m}^{-1}$. The reflected beam at the forcing frequency, which radiates away from the pycnocline into the lower layer, is in stark contrast with the higher harmonics $2 \omega_{f}$ (and $3 \omega_{f}$, not shown in the figure), which are trapped in the upper part of the water column and propagate purely horizontally with a well-defined horizontal periodicity. For the $2 \omega_{f}$-signal, we find a horizontal wavelength of $22 \mathrm{~cm}$. The fact that the harmonics are trapped is readily understood from linear internal-wave theory, since their frequencies $2 \omega_{f}, 3 \omega_{f}$ etc. exceed the buoyancy frequency of the lower layer $\left(N_{0}\right)$; hence the harmonics cannot propagate into it and are constrained to the pycnocline.

The experimentally measured horizontal wavelength of the trapped higher harmonics may be compared with the theoretical estimates from the eigenvalue problem for the 


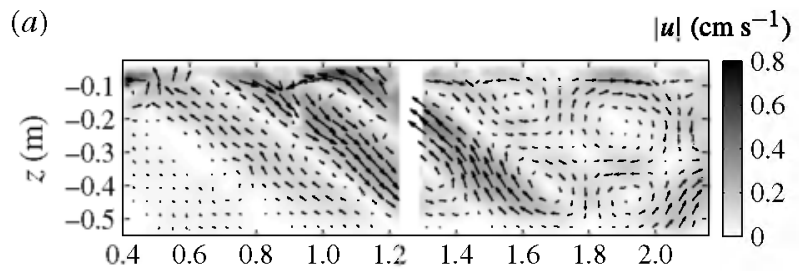

(b)
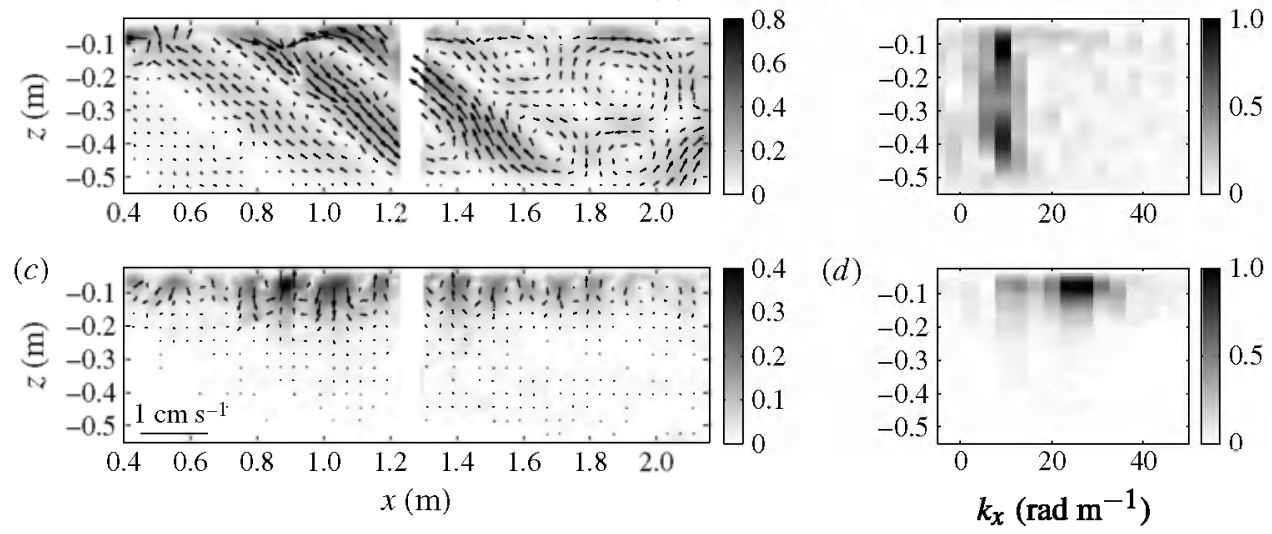

FIGURE 5. EXP25. Harmonic analysis for the forcing frequency $\omega_{f}(a, b)$, and the first harmonic $2 \omega_{f}(c, d) .(a, c)$ The velocity vector field and its amplitude. The arrow indicating the scale of the velocity vectors in $(c)$ is also valid for $(a) .(b, d)$ The horizontal Fourier spectra of the vertical velocity $w$ as a function of the horizontal wavenumber $k_{x}$ and its vertical variation (for each, amplitudes are normalized by the maximum value).

vertical modal structure

$$
W^{\prime \prime}+\left[\frac{N^{2}}{(U-c)^{2}}-\frac{U^{\prime \prime}}{U-c}-k^{2}\right] W=0,
$$

with the vertical velocity $w(x, z, t)=\operatorname{Re}[W(z) \exp [\mathrm{i}(k x-\omega t)]]$, and the boundary condition $W=0$ at the surface and bottom (LeBlond \& Mysak 1978, equation (41.8)). Here, $c=\omega / k$ is the horizontal phase speed, $U(z)$ is the background mean flow profile, $U^{\prime \prime}(z)$ its second derivative and $N(z)$ the buoyancy frequency profile. We note that this equation is different from the one in terms of the vertical isopycnal displacement, $\eta$. In the literature, the two forms are sometimes confused. The relation between the two follows from $w=\eta_{t}+U \eta_{x}$, so that $W=\mathrm{i} k(U-c) \phi$, where $\eta=\operatorname{Re}\{\phi(z) \exp [\mathrm{i}(k x-\omega t)]\}$.

For $\omega=2 \omega_{f}$, and observed $N(z)$ and $U(z)$, we solve (3.1) to obtain the modes $W_{n}$ and corresponding wavenumbers $k_{n}$ or wavelengths $\lambda_{n}=2 \pi / k_{n}$. For EXP25, we find $\lambda_{1}=48.9, \lambda_{2}=20.2$ and $\lambda_{3}=14.8 \mathrm{~cm}$, using the background mean flow profile measured at $x=1.0 \mathrm{~m}$. The observed horizontal wavelength (i.e. $22 \mathrm{~cm}$ ) of the signal at $\omega=2 \omega_{f}$ is the closest to that of the theoretical mode 2 . This is confirmed by the excellent agreement between the experimental and theoretical profiles of $W_{2}$ and $U_{2}$, as shown in figure 6, at least so far as the comparison can be made (as mentioned earlier, PIV data is not available in the upper few centimetres). Exactly why the first harmonic (i.e. $2 \omega_{f}$ ) manifests itself as a second mode, and not as some other mode, is not clear. For instance, we note that the horizontal phase speed of the incident internal wave beam, $1.8 \mathrm{~cm} \mathrm{~s}^{-1}$, is not particularly close to either the measured $\left(2.6 \mathrm{~cm} \mathrm{~s}^{-1}\right.$ ) or theoretically calculated $\left(2.4 \mathrm{~cm} \mathrm{~s}^{-1}\right)$ mode 2 phase speed for $\omega=2 \omega_{f}$. In fact, it is closest to the theoretically calculated horizontal mode 3 phase speed for $\omega=2 \omega_{f}$ $\left(c_{3}=2 \omega_{f} / k_{3}=1.8 \mathrm{~cm} \mathrm{~s}^{-1}\right)$. This suggests that a phase-speed-matching mechanism, such as the one discussed by Grisouard et al. (2011), who examined the local generation of internal solitary waves with a numerical model but without observing any mean flow, is not in play here. Two possible explanations may be advanced. In a weakly nonlinear case where there is just advection of the harmonics by the mean 


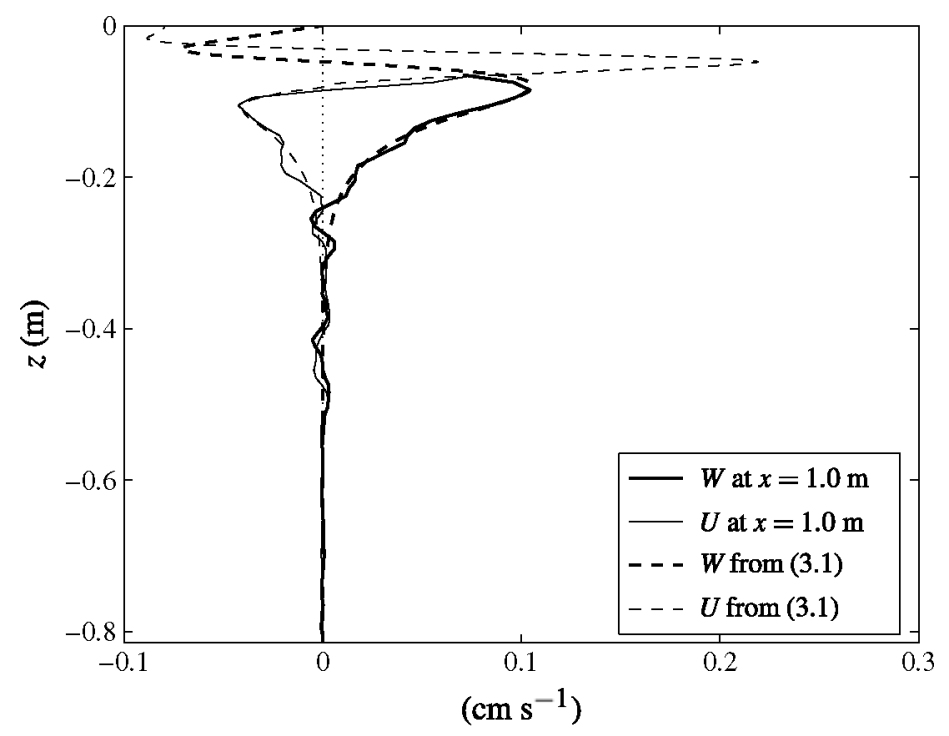

FIGURE 6. EXP25. Comparison between the mode 2 shape from experimental velocity profiles at $x=1.0 \mathrm{~m}$, filtered at $\omega=2 \omega_{f}$, and those calculated from (3.1).

flow, we could search for a criterion with Doppler effect on the phase speed of the harmonics. Another more complex scenario corresponds to fully nonlinear coupling of the mean flow and the harmonics. However, our experiments cannot provide an answer to this point of high interest.

Finally, the importance of the shear flow to the modal propagation of the higher harmonics can be illustrated by solving the modes from (3.1) with $U=0$. Then, for $\omega=2 \omega_{f}$, we find much smaller horizontal wavelengths: $\lambda_{1}=41, \lambda_{2}=13$ and $\lambda_{3}=8 \mathrm{~cm}$, none of which matches the observed wavelength.

For EXP22 $\left(\omega_{f} / N_{0}=0.50\right)$, we again find that the measured horizontal wavelength (35 cm) of the harmonic $2 \omega_{f}$ is closest to the corresponding theoretical wavelength of mode $2\left(\lambda_{1}=74, \lambda_{2}=29\right.$ and $\left.\lambda_{3}=20 \mathrm{~cm}\right)$. We note, however, that the wavelengths are in this case $\left(2 \omega_{f} \approx N_{0}\right)$ sensitively dependent on the frequency $\omega=2 \omega_{f}$ and $N_{0}$, because they are very close. For example, the mode 2 wavelengths for $\omega=0.5$ and $0.6 \mathrm{rad} \mathrm{s}^{-1}$ for the stratification in EXP22 are $\lambda_{2}=73$ and $24.5 \mathrm{~cm}$, respectively. Thus the quantitative agreement between experiment and theory could be better or worse for even small changes in $\omega$ or $N_{0}$. As in the case for EXP25, the vertical structure of the first harmonic signal $\left(2 \omega_{f}\right)$ agrees well with the theoretically computed mode 2 shape, except in the deep parts of the lower layer. In the lower layer, where the measured background shear flow is negligible, (3.1) predicts a linear variation of $W$ with $z$ for $\omega=2 \omega_{f} \approx N_{0}$ (since the equations boil down to $W^{\prime \prime} \sim 0$ ); however, we observe a more rapid decay of the signal in the lower layer, the cause of which remains unclear.

In the case of EXP21 $\left(\omega_{f} / N_{0}=0.26\right)$, for which $3 \omega_{f}<N_{0}$, the first two harmonics are not trapped but are allowed to propagate into the lower layer; hence they radiate from the pycnocline as beams and cannot be identified with any one mode, as shown in figure 7. However, a different simple regularity appears from figure 7. For each harmonic $(n)$, the beam pattern is clearly periodic (see figure $7 a, c, e$ ); the horizontal wavenumber associated with this periodicity $\left(k^{(n)}\right.$, say) obeys the simple 
(a)
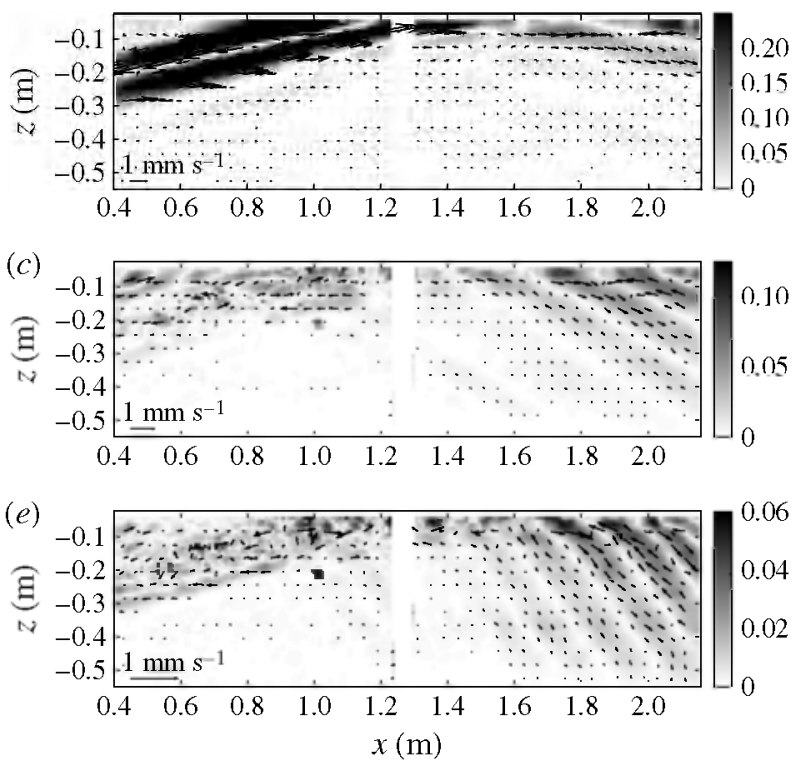

(b)

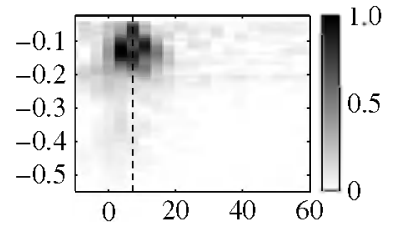

(d)
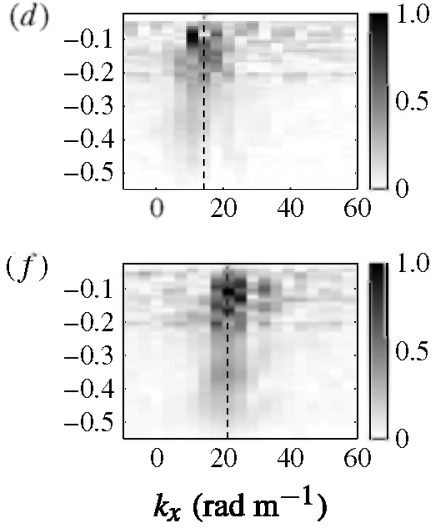

FIGURE 7. EXP21. Harmonic analysis as in figure 5 for the frequency $n \omega_{f}$, with $n=1,2,3$. The dashed lines in $(b, d, f)$ represent, for harmonic $\omega=n \omega_{f}$, the value $k^{(n)}=n k^{(1)}$ with $k^{(1)}=7.1 \mathrm{rad} \mathrm{m}^{-1}$.

rule $k^{(n)}=n k^{(1)}$ (see dashed lines in figure $7 b, d, f$ ). This implies that the horizontal phase speeds are the same for all harmonic beams.

Finally, it is evident from figures 5 and 7 that the amplitude of the harmonics at $n \omega_{f}$ is weaker than the one at $(n-1) \omega_{f}$ (for $\left.n>1\right)$, and that these higher harmonics originate from the region of interaction between the incident wave beam and the pycnocline. These observations confirm that the higher harmonics are a result of weakly nonlinear interactions between the incoming and reflected beams. Since the Reynolds number varies along with other parameters through the experiments (cf. table 1), we are unable to determine its role in the generation of harmonics, more specifically its effect on the saturation of the harmonics amplitude, as already observed by King et al. (2009) for instance. Varying the forcing frequency across various experiments results in the value of $\omega_{f} / N_{0}$ go from values smaller to larger than 0.5 , and thus influencing the propagation in the lower constant stratification layer of higher harmonics; freely propagating for $\omega_{f} / N_{0}<0.5$ and trapped in the pycnocline for $\omega_{f} / N_{0}>0.5$. The change in the ratio $\omega_{f} / N_{\max }$, however, remains weak and does not seem to play any role in our experiments. A detailed study of the influence of the amplitude of the incoming beam on the amplitude of the generated harmonics is recommended for future research.

\section{Evolution of the pycnocline}

In this section, we discuss the evolution of the pycnocline, as measured by the acoustic probes. As mentioned in $\$ 2.4$, ten acoustic probes were positioned at various positions in the range $0-4 \mathrm{~m}$ from the wave generator. The proximity of two probes located at $x=1.20$ and $1.254 \mathrm{~m}$, respectively, allows us to determine the horizontal 
(a)

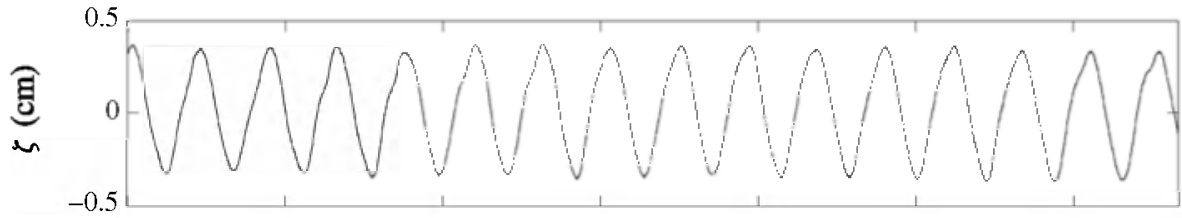

(b)

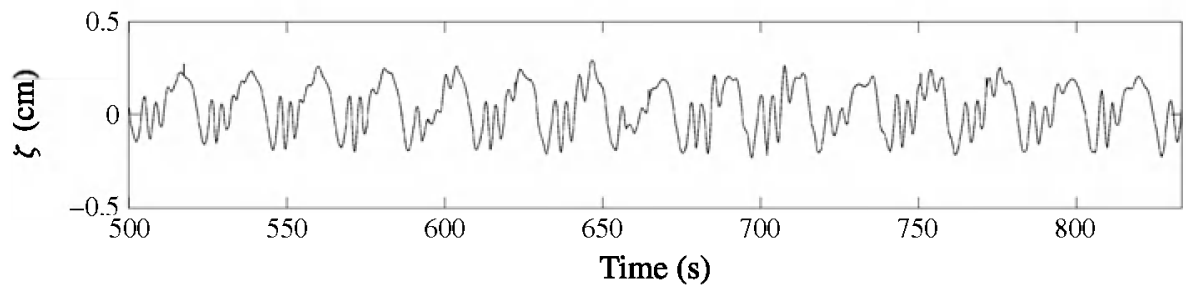

(c)

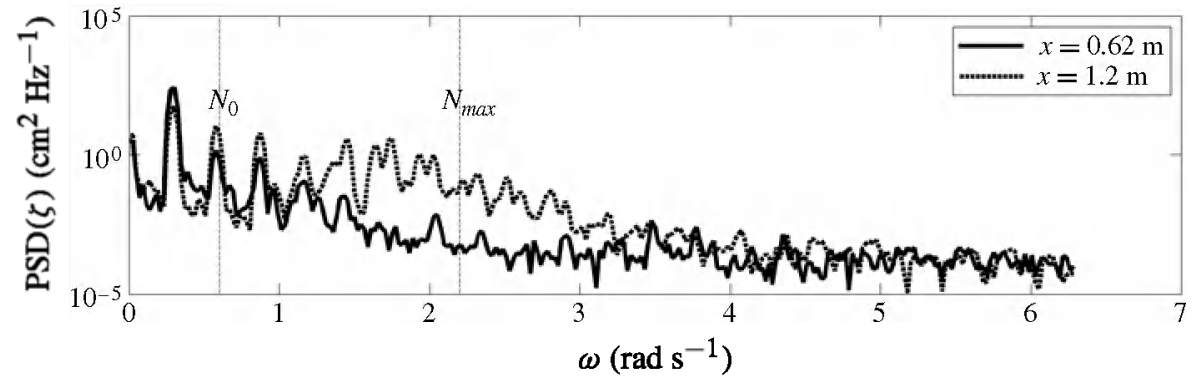

FIGURE $8 . \operatorname{EXP08}(\gamma=0.11)$. Steady-state pycnocline displacements at $(a) x=0.62 \mathrm{~m}$ and (b) $x=1.2 \mathrm{~m}$. (c) Corresponding Fourier spectra.

phase speed of the signal, for its structure remains similar between the two, so that specific peaks can be identified in both. In every experiment, the acoustic probe measurements were initiated well before the wave generator was started, allowing us to visualize the transients before a steady state was reached.

In figure $8(a, b)$, we plot the pycnocline displacements $\zeta$ (i.e. vertical averages over the thickness of the pycnocline, as described in $\S 2.4$ ) at $x=0.62 \mathrm{~m}$ and $x=1.2 \mathrm{~m}$, respectively. In a qualitative way, the results from the probes indicate the formation of ISWs in the pycnocline: the wave pattern is nearly sinusoidal at the region of impact $(x=0.62 \mathrm{~m})$, steepens as it propagates away, and at some point its steep front splits up into higher-frequency peaks, which are, at least initially $(x=1.2 \mathrm{~m})$, ordered in amplitude, the larger ones being ahead. Furthermore, as shown in figure $8(\mathrm{c})$, the power spectrum of the time series at $x=1.2 \mathrm{~m}$ has a distinct bulge around the frequencies in the neighbourhood of $\omega=N_{\max }$, a feature we discuss in detail later in the current section. In a quantitative sense, however, it is not obvious that nonlinear effects are at work; after all, the amplitude of the peaks is small (a few millimetres) compared to the depth of the pycnocline (a few centimetres).

We note, however, that the actual peak amplitudes of interfacial displacements must be larger than the measured outputs from the ultrasonic probes as the probes measure the mean amplitudes over the layer between the transmitter and the receiver. This effect is particularly pronounced for the high-frequency waves that are trapped in the pycnocline, for their amplitude diminishes rapidly outside of the pycnocline. We also note that the shape and amplitude of the ISWs we find are similar to those observed in earlier experiments on internal solitons in a two-layer system (Horn, Imberger \& Ivey 2001). 


\subsection{Interpretation of spectra}

The generation of higher harmonics due to nonlinear interaction at the junction of two beams was discussed in $\$ 3.2$. Being relatively weak, their behaviour must be close to linear as they propagate away from their source. This means that they can be properly identified in spectra, as seen in figure $8(\mathrm{c})$, which after all amounts to treating the signal as if it were linear. For ISWs it is less clear how they might manifest themselves in spectra. Being genuinely nonlinear waves, they are not associated with any particular harmonic, nor can they be described as a superposition of independently propagating harmonics. Still, technically, one can calculate the spectrum of a signal containing ISWs. As a test case, we use results from a set of KdV-type equations for interfacial waves, which include forcing terms due to barotropic tidal flow over topography (Gerkema 1996). The model is weakly non-hydrostatic and weakly nonlinear; besides, it contains terms describing the advection of baroclinic velocity fields by the barotropic flow. In one model calculation, we switched off the nonlinear terms while retaining the barotropic advection terms. In this case, the internal-wave field is linear but contains higher harmonics due to the barotropic advection terms. In another calculation, the nonlinear terms were included, giving rise to internal solitons. In this case, there are higher harmonics as well as ISWs, and so this is qualitatively the situation we supposedly have in our laboratory experiments.

If we now calculate the spectra for the results of both model runs, we find important and qualitative differences (see figure 9). For the quasi-linear case, peaks at higher harmonics are found, and, as expected, they get lower and lower for higher frequencies. In the nonlinear case, the same qualitative behaviour is found at the lowest frequencies, but then a bulge appears, which is not associated with any one harmonic, but more widespread, involving a group of harmonics. This is easily understood from the fact that solitons are not associated with harmonics per se, but nonetheless have certain time scales within the range of some of the harmonics. Harmonics in the neighbourhood of these time scales show marked peaks in the spectrum. This does not mean that the solitons are formed by a superposition of independently propagating higher harmonics (after all, the signal is nonlinear), but are the result of applying a linear technique (harmonic analysis) to a nonlinear signal. In any case, the presence of a bulge helps to distinguish the physically distinct cases of pure higher harmonics on the one hand, and solitons on the other.

Such a bulge appears in our experiments too, as is illustrated in figure $8(\mathrm{c})$. This confirms the direct visual impression from the probe results shown in figure $8(b)$, in which groups of peaked waves are clearly visible. We proceed, in the next section, to investigate the effect of the pycnocline properties (depth, vertical extent, strength etc.) on the bulge, and hence propose a way to capture all the properties into one parameter, the magnitude of which determines the appearance of solitons.

\subsection{Effect of the pycnocline strength}

By removing the upper layer and refilling it with fresh water, we varied the depth, the amplitude, and the width of the peak of the pycnocline (see figure $10 \mathrm{a}$ for the various stratification profiles that were set up during the course of all our experiments). In this section, we present results from three experiments with the same forcing frequency (such that the incident internal wave beam propagates at $30^{\circ}$ with respect to the horizontal) but with different pycnocline properties.

A key parameter for the response of the pycnocline, due to an impinging internalwave beam, was identified by Gerkema (2001) using linear theory. In that study, a ' $2 c$ layer' stratification was adopted, consisting of an upper mixed layer of thickness $d$, an 


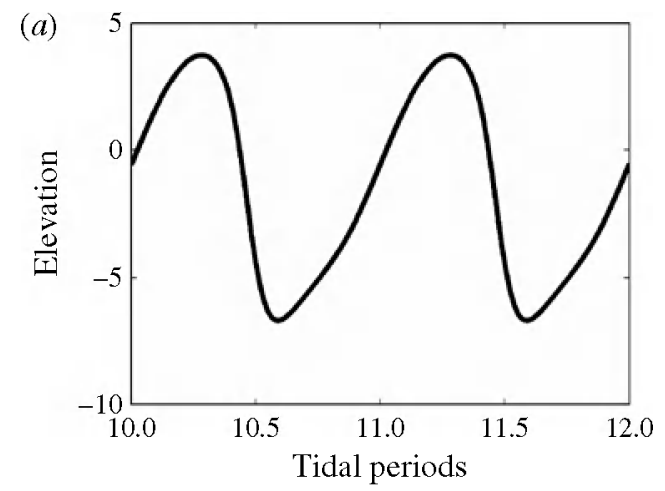

(b)
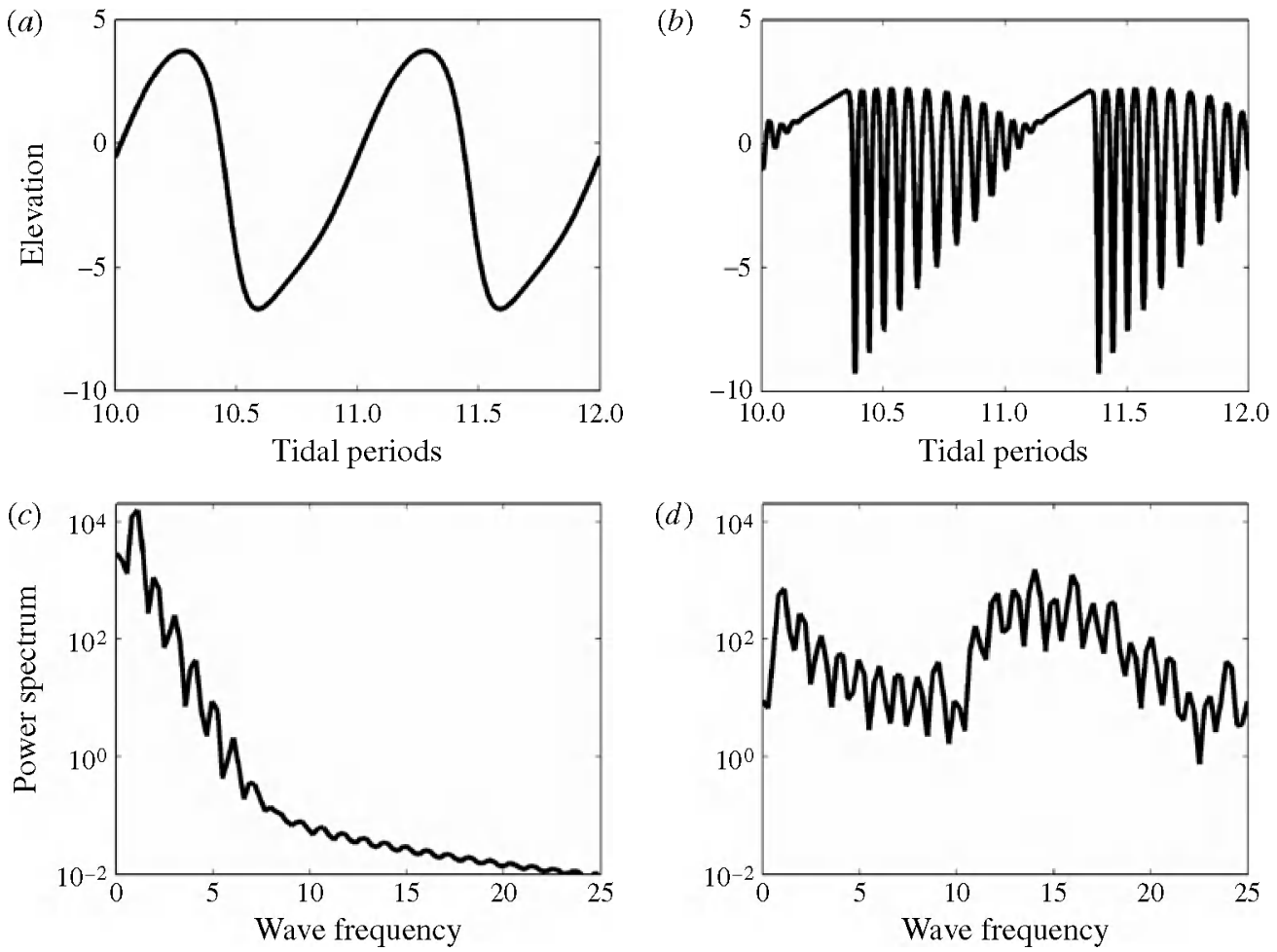

(d)

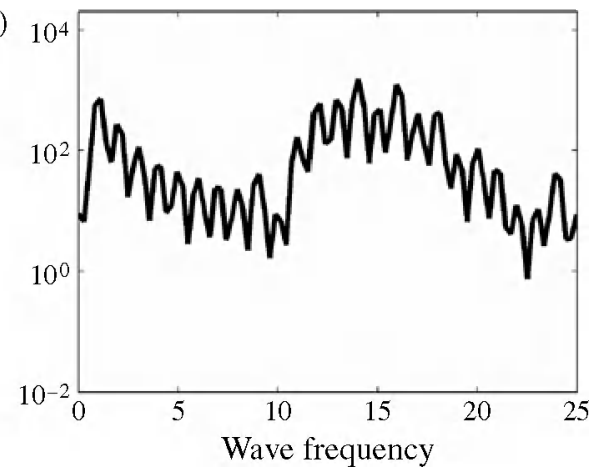

FIGURE 9. Examples from a nonlinear non-hydrostatic KdV type model for interfacial tidally generated waves. (a) The wave profile over two tidal periods, created from the quasi-linear version of the model, where higher harmonics are generated by barotropic advection of the baroclinic field; these harmonics manifest themselves as peaks in the corresponding power spectrum, shown in $(c)$. In $(b)$, genuinely nonlinear effects of the baroclinic field itself are included, giving rise to solitons; the corresponding spectrum in $(d)$ is now markedly different, showing a bulge enveloping higher frequencies.

interfacial pycnocline of strength $g^{\prime}$, beneath which lies a layer of constant buoyancy frequency, $N_{c}$. If the total water depth is $H$, the parameter in question is defined by

$$
\gamma=\frac{\left(g^{\prime} d\right)^{1 / 2}}{N_{c} H} .
$$

In the numerator of (4.1), we recognize the phase speed of long interfacial waves in a two-layer system, while the expression in the denominator is a measure of the phase speed of vertical modes in a constantly stratified layer of depth $H$. These modes add up to form beams, so the parameter $\gamma$ reflects the ratio of the phase speeds associated with the interface (i.e. pycnocline) and the uniformly stratified lower layer. It was found by Gerkema (2001) that for $\gamma$ either very small or very large, there is no significant transfer of energy from beams to interfacial waves. Such a transfer occurs only for intermediate values, characterized by $\gamma \approx 0.1$. This would therefore be expected to serve as a necessary condition for having locally generated ISWs which emerge from the initial interfacial perturbation as it propagates and steepens. It is, however, not a sufficient condition, for the forcing frequency, which does not feature in (4.1), must also affect the evolution into ISWs. 

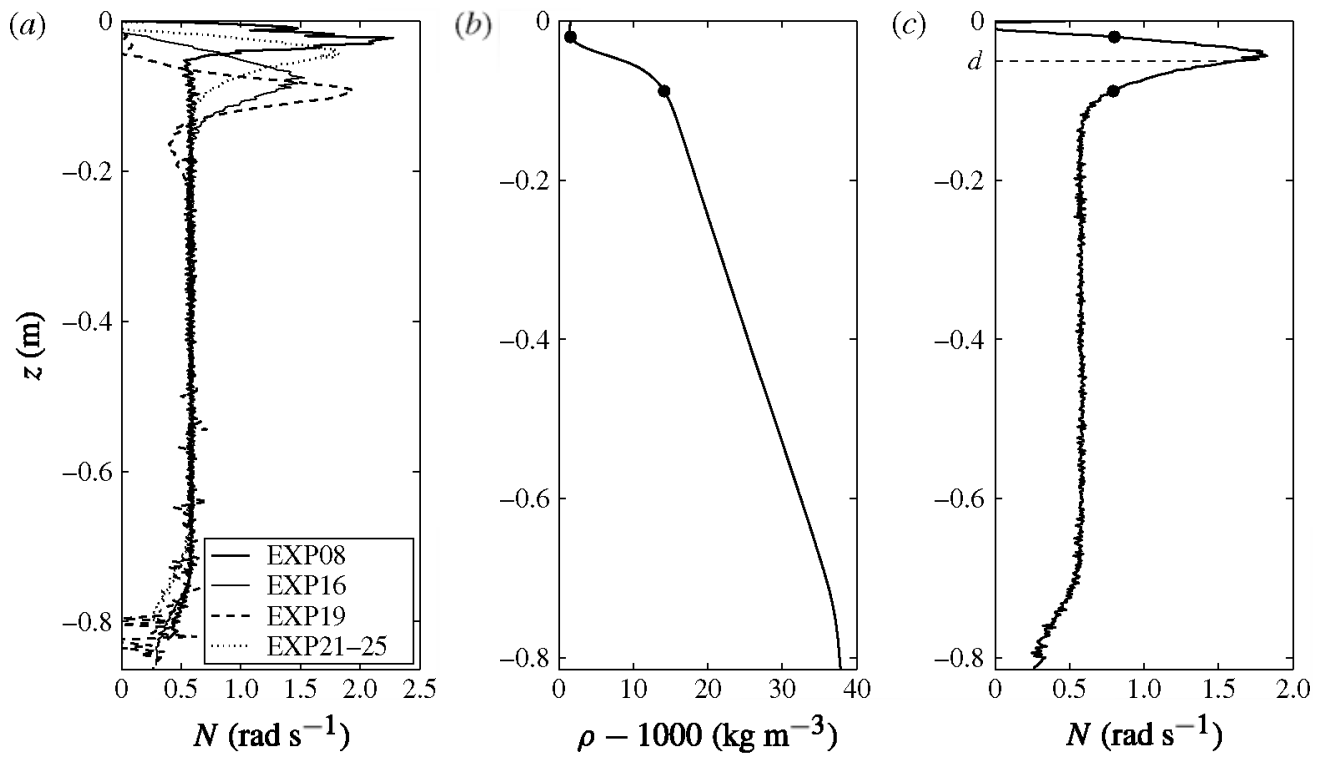

FIGURE 10. (a) Different profiles of stratification with which experiments were carried out. $(b, c)$ Determination of $\gamma$ from the profile for experiments EXP21-25. The black dots indicate the (approximate) positions of the top and base of the pycnocline, delineating the vertical interval where density changes strongly in $(b)$. The depth of the weighted centre of the pycnocline, i.e. $d$, is indicated by the horizontal dotted line in $(c)$.

In the present situation we have a more complicated profile of stratification, but the parameters in (4.1) are still meaningful if interpreted in the following way. First, we look at the density profile to identify the base and top of the pycnocline; an example is shown in figure $10(b, c)$. As explained in Gerkema (2001), the parameter $g^{\prime}$ equals the area enclosed by the pycnocline in the $N^{2}$ versus $z$ graph; so, $g=\int N^{2} \mathrm{~d} z$, where the integral is taken between the previously identified base and top of the pycnocline. The parameter $d$ corresponds to the depth of the pycnocline, but this depth is of course not uniquely defined here. To take the depth of the peak would not always make sense because it may not be representative of where the bulk of the pycnocline lies. Therefore, we calculated a 'weighted depth', as $d=\int z N^{2} \mathrm{~d} z /\left(\int N^{2} \mathrm{~d} z\right)$, where the boundaries of the integrals are again the base and top of the pycnocline. The depth $d$ thus found is illustrated in figure $10(c)$. Finally, the parameter $H$ is simply the total water depth, and $N_{c}$ is taken equal to the mean of $N$ from the bottom to the base of the pycnocline. We have thus obtained all the parameters needed to calculate $\gamma$, which is listed for different profiles in figure $10(a)$ and also in table 1.

In figure $11(a-c)$, the pycnocline displacements at $x=1.59 \mathrm{~m}$ from three different experiments (all corresponding to $\omega_{f}=0.29 \mathrm{rad} \mathrm{s}^{-1}$ ) with $\gamma=0.11,0.17,0.19$, respectively, are presented. As is evident from the figures, groups of peaked waves are most clearly visible for $\gamma=0.11$ (figure $11 a$, EXP08). The corresponding power spectra, presented in figure $12(a-c)$, show that the bulge at frequencies larger than the forcing frequency is most pronounced for EXP08, confirming that the bulges in the power spectra are indeed directly correlated with the appearance of solitons. The bulge is relatively weaker in EXP22, and is the weakest in EXP16, for which the higher 

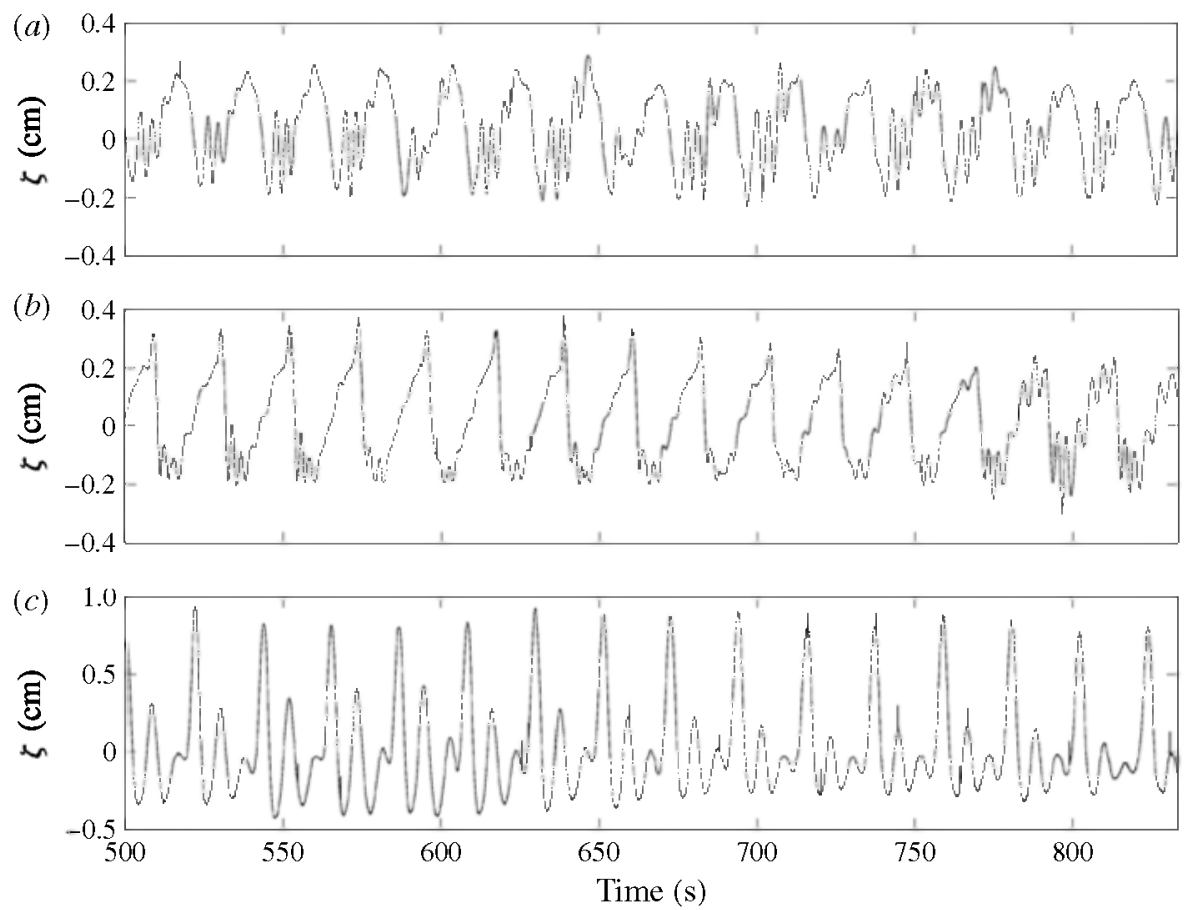

FIGURE 11. Steady-state pycnocline displacements at $x=1.59 \mathrm{~m}$ in $(a) \operatorname{EXP0} 0(\gamma=0.11)$, (b) $\operatorname{EXP22}(\gamma=0.17)$, and (c) $\operatorname{EXP16}(\gamma=0.19)$.

harmonics dominate over solitary-wave-like features. Finally, the solitons being most evident for $\gamma=0.11$ is consistent with the theoretical criterion of $\gamma \approx 0.1$ given by Gerkema (2001) for optimal excitation of solitons in the pycnocline, and also with oceanic observations. Indeed, recent studies of the stratification in the Mozambique Channel, where local generation of ISWs was proposed by Da Silva et al. (2009), give $\gamma=0.08$, a value fairly close to the observed optimum stratification for soliton generation in our experiments.

The two closely spaced probes at $x=1.2 \mathrm{~m}$ and $x=1.254 \mathrm{~m}$ were introduced only after EXP08 was performed. Analysing the time series data from these two closely spaced acoustic probes in $\operatorname{EXP} 22\left(2 \omega_{f} \approx N_{0}\right.$ and $\left.\gamma=0.17\right)$, we find that the higher-frequency peaks move with the same speed as the main depression (or the envelope) on which they sit. They move at $2.3 \mathrm{~cm} \mathrm{~s}^{-1}$, the speed being larger than the mode 1 wave speed $\left(c_{1}=1.6 \mathrm{~cm} \mathrm{~s}^{-1}\right.$, calculated by numerically solving (3.1) with the measured $N(z)$ and $U(z)$ at $x=1.15 \mathrm{~m}$ ) for $\omega=2.5 \mathrm{rad} \mathrm{s}^{-1}$ (frequency representative of the bulge observed in figure $12 b$ ). This suggests that the observed solitary waves were mode 1 , with the solitons moving faster than the corresponding linear mode 1 wave because of nonlinear effects. Finally, the soliton speed is neither very close to the horizontal phase speed of the incident wave beam $\left(1.83 \mathrm{~cm} \mathrm{~s}^{-1}\right.$, derived from the PIV data, again ruling out the phase-speed-matching mechanism in our experiments) nor close to that of the second-mode structure of the the second harmonic discussed in $\S 3$ (with observed $\lambda_{2}=35 \mathrm{~cm}$, we obtain $3.24 \mathrm{~cm} \mathrm{~s}^{-1}$, suggesting that the solitons are fairly independent from the higher harmonics). 


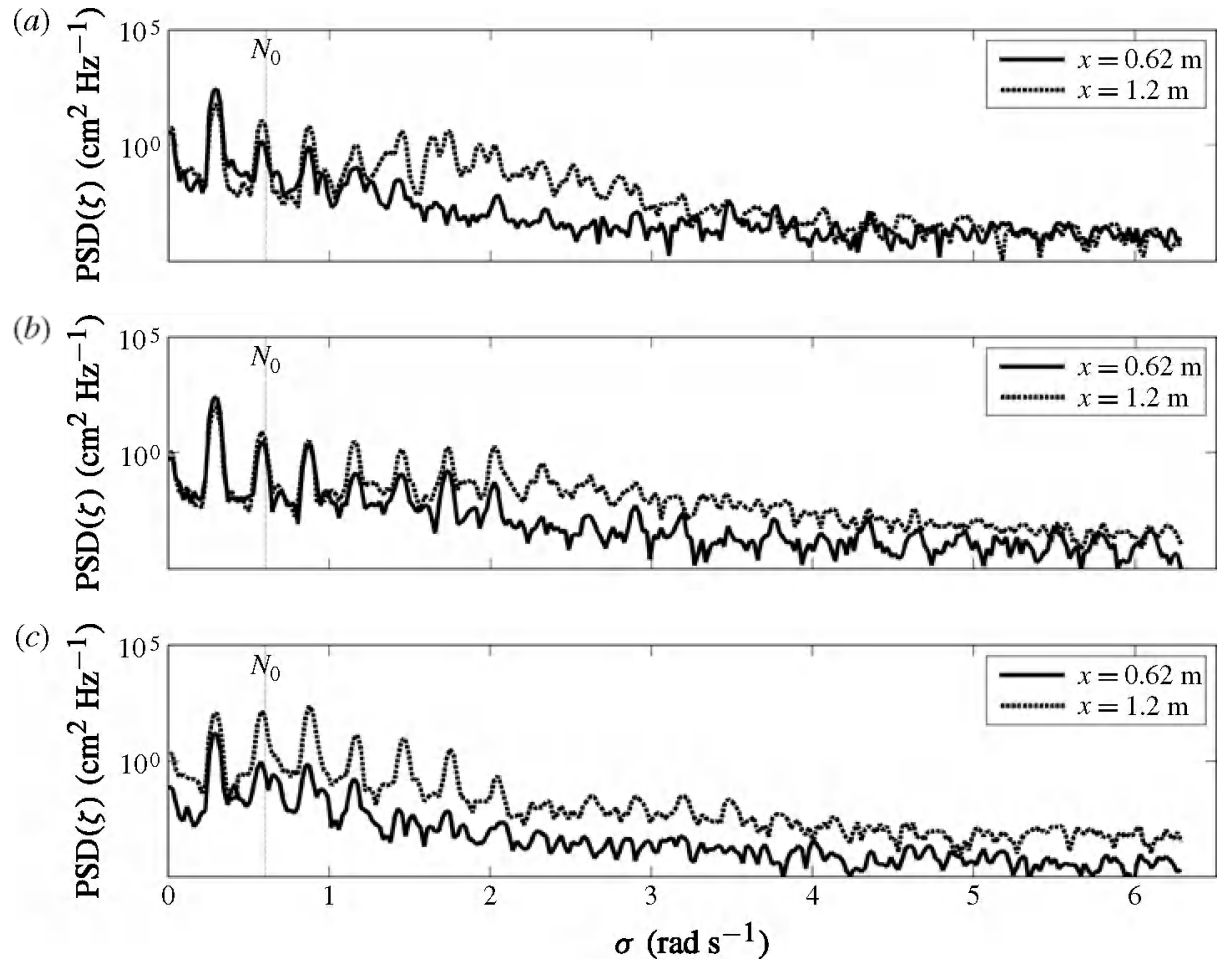

FIGURE 12. Power spectra of the steady-state pycnocline displacements at $x=0.62 \mathrm{~m}$ and $x=1.20 \mathrm{~m}$, in $(a) \operatorname{EXP08}(\gamma=0.11),(b) \operatorname{EXP} 22(\gamma=0.17)$, and $(c) \operatorname{EXP16}(\gamma=0.19)$, with all the three plots normalized by the same constant.

\section{Discussion}

Our experiments were inspired by oceanic observations (New \& Pingree 1992; New \& Da Silva 2002; Azevedo, Da Silva \& New 2006; Da Silva et al. 2007, 2009), theoretical works (Gerkema 2001; Akylas et al. 2007) and numerical studies (Maugé \& Gerkema 2008; Grisouard et al. 2011). Apart from the expected result of the generation of groups of solitary waves, the full picture that emerges turns out to be intricate, with the additional features of a mean current and higher harmonics.

We used two measurement techniques, which were complementary. PIV measurements were particularly suitable for obtaining data on the harmonics and mean flow in the layer beneath the pycnocline. Ultrasonic probes were used to measure the vertical movement of the pycnocline itself. At the base of the pycnocline there is an overlap: both techniques provide reliable measurements in this region. A discussion of the consistency between the two techniques is presented in appendix $\mathrm{A}$.

We have found higher harmonics being generated at the junction of the incident and reflected beams, as expected from theory by Tabaei \& Akylas (2003). Moreover we found that these harmonics are trapped in the pycnocline when their frequency exceeds or is close to that of the constantly stratified lower layer. These trapped harmonics are each uni-modal, with a well-defined wavelength. The wavelengths and vertical profiles of these modes are significantly affected by a residual current in the upper layer, which, too, finds its origin in the region where the main beam reflects. Theoretical studies of internal-wave reflection do predict residual currents (Tabaei et al. 2005, 

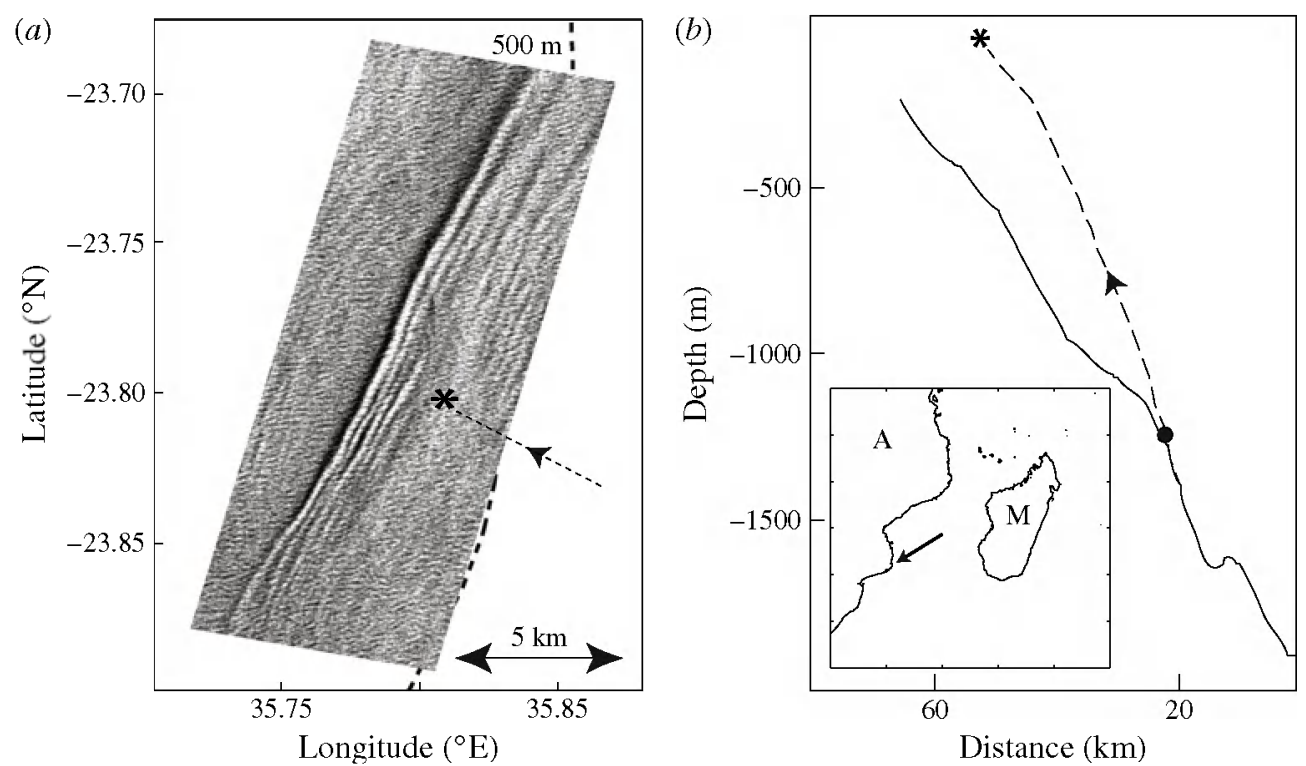

FIGURE 13. (a) SAR image of the Mozambique Channel dated 24 September 2001 at 7:39 UTC, showing a packet of ISWs consistent with mode 2 vertical structure. The star indicates the predicted position where an internal tide beam impinges the thermocline. (b) On the right-hand side a vertical section (perpendicular to the ISW crests) is shown with a simulated beam trajectory based on local stratification (represented by the dashed line). The black circle indicates critical slopes from where the beam seemingly originates. The star shows the location where the beam impacts the thermocline (as well as in part $a$ ), and the arrows indicate the direction of energy propagation. The inset shows the geographical location of the observation; $M$ stands for Madagascar and A for the African continent (the arrow in the inset denotes the location of the image).

for example), but only in the actual region of reflection. Our experiments, however, demonstrate that they can be much more extensive horizontally, in the direction of wave propagation. We are not aware of any theoretical work that can explain this phenomenon. The importance of the mean flow on the generation of harmonics, and also the apparently three-dimensional structure of the flow, both deserve separate studies.

We observe the generation of internal solitary waves only when the stratification is such that the parameter $\gamma$ is close to 0.1, which is consistent with the theoretical criterion put forward by Gerkema (2001). We have demonstrated that these waves, although weak in amplitude, are intrinsically nonlinear, as they manifest themselves in spectra not as a superposition of harmonics but as a bulge. This interpretation of the spectra is suggested by looking at spectra from a KdV-type model. It is also to be noted that we did not perform experiments at non-zero values smaller than $\gamma=0.11$. We therefore suggest investigations in this regime in future experiments to conclusively establish the optimality of $\gamma=0.1$ for the generation of solitary waves.

Although our experiments clearly show the generation of trapped mode 2 higher harmonics in the pycnocline, the ISWs seem to be of mode 1, in line with most oceanic observations. Yet, exceptions may occur, as is clear from the numerical work by Grisouard et al. (2011) and also from oceanic observations such as that shown in figure 13. Here we see a SAR image from the Mozambique Channel with 
surface signatures of ISWs. While these signatures look like many other typical SAR signatures of ISWs, with larger-amplitude waves leading the wave trains in their direction of propagation, close inspection of their bright and dark patterns reveals that they are mode 2 solitary-like waves, as explained in appendix B. Indeed, in this region of $500 \mathrm{~m}$ depth with a pycnocline typically resting at $100 \mathrm{~m}$ below the free surface (Da Silva et al. 2009), a dark band pattern preceding a bright band (in the propagation direction) reveals a surface current divergence preceding a convergence, which is consistent with a mode 2 solitary-like wave (Da Silva, New \& Magalhaes 2011). In addition, the ISWs in figure 13 are located close to (but ahead of) the surfacing of an internal (tidal) ray emanating from critical slopes off the Mozambique shelf break. The image in figure 13 provides evidence of mode 2 solitary-like waves that are consistent with local generation after the impact of an internal (tide) wave beam on the pycnocline.

As mentioned in $\S 1$, it was not possible for us to make a direct comparison between our experiments and the numerical simulations of Grisouard et al. (2011). The mean flow observed in our experiments, discussed in $\S 3$, was not observed in the numerical studies. Since the mean flow affects the phase speeds of the modes, it cannot be established from the numerical study how this might affect the criterion for mode selection proposed by Grisouard et al. (2011). The same is true for the higher harmonics. So, our laboratory experiments are an inspiration for a next step in the numerical modelling on local generation: especially to study the origin and effect of a mean flow.

Finally, we are not aware of oceanic observations in which trapped higher harmonics were found in a pycnocline. In the context of internal tidal beams impinging on a seasonal thermocline, this phenomenon is in any case not be expected, for the semidiurnal tidal frequency $\left(1.4 \times 10^{-4} \mathrm{rad} \mathrm{s}^{-1}\right)$ is much lower than the typical value of $N$ in the seasonal thermocline $\left(1 \times 10^{-2} \mathrm{rad} \mathrm{s}^{-1}\right)$; in other words, the harmonic would have to be of an extremely high multiple for it to be trapped. The mean flow, on the other hand, has not been observed either, as far as we are aware, but there seems to be no a priori reason why it should not occur. In any case, in oceanic observations, too, one would typically find a mixture of higher harmonics (not trapped) and internal solitary waves. The idea we have put forward here to distinguish them in internal-wave spectra may provide a useful tool in the oceanographic context as well.

\section{Acknowledgements}

We thank S. Viboud and H. Didelle for their help during the experiments, M. Moulin for the cams design and acknowledge helpful discussions with J. Sommeria. We also thank the anonymous referees for useful suggestions concerning the mean flow. The experiments were supported by funds from the Hydralab III Transnational Access Program (6th FP) and ANR PIWO (contract number ANR-08-BLAN-0113). We also thank MIT-France for partially funding M.M.'s travel expenses.

\section{Appendix A. Comparing PIV and ultrasonic measurements}

Two different techniques were used to study the internal wave beam impinging on a pycnocline. PIV data from the lower layer of constant stratification provide velocity fields in a vertical plane, with a sampling frequency of $3 \mathrm{~Hz}$. An array of ultrasonic probes, on the other hand, gives time series of local mean vertical displacements of the pycnocline at a much higher sampling frequency of $240 \mathrm{~Hz}$. The two techniques are complementary since the PIV measurements offer a large field of view and the 

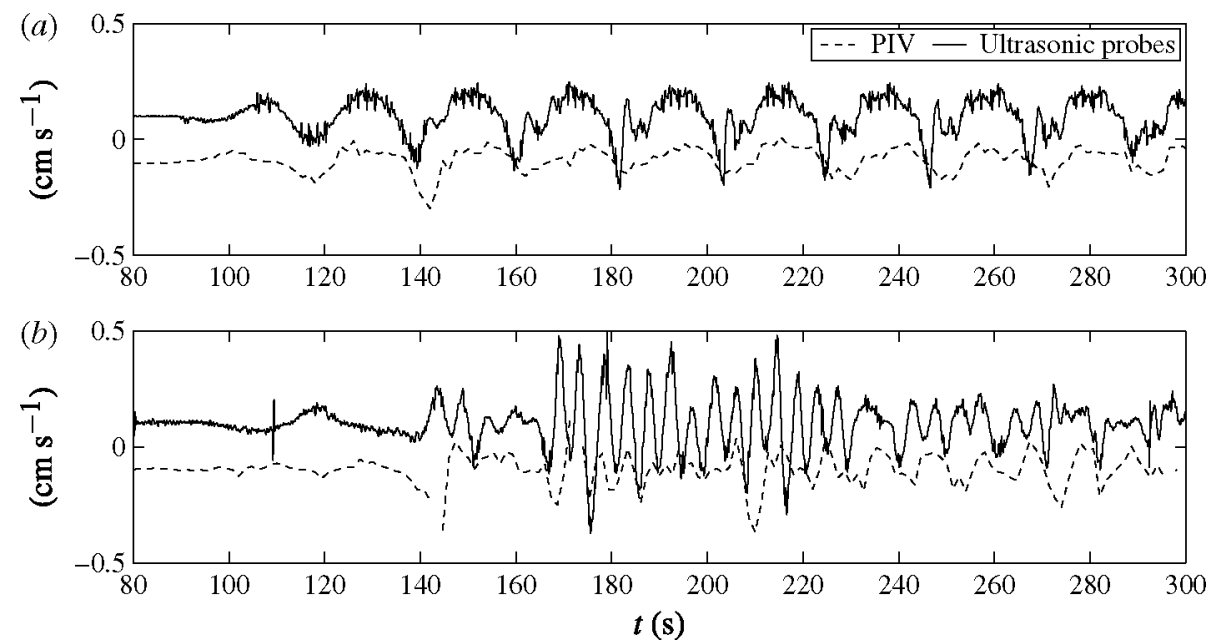

FIGURE 14. EXP22. Comparison of time series of the vertical velocity $\mathrm{d} \zeta / \mathrm{d} t$ extracted from the ultrasonic probes and from the PIV images at the closest location to the ultrasonic probes, for probes located at $(a) x=0.62 \mathrm{~m}$ and $(b) x=1.20 \mathrm{~m}$. The data do oscillate around 0 but are arbitrarily shifted by $\pm 0.1 \mathrm{~cm} \mathrm{~s}^{-1}$ to make the comparison easier.

ultrasonic probes focus on the pycnocline, where PIV cannot be used because of strong optical distortion and sparse particle seeding. They can also be compared to a certain extent and hence be used to reaffirm the conclusions drawn from the data and to demonstrate their consistency.

The first time-derivative of the mean pycnocline displacements measured by the ultrasonic probes should qualitatively correspond to the vertical velocity obtained from PIV data from just below the pycnocline. The comparison can only be qualitative because the acoustic probes provide a vertical average of displacements within the pycnocline, not a value at a specific depth. To illustrate this point, we present in figure 14 time series of the vertical velocity of the pycnocline obtained with the two techniques at $x=0.62 \mathrm{~m}$ and $x=1.2 \mathrm{~m}$ for EXP22. The continuous lines are obtained by taking the time derivative of the probe signals; the dashed lines represent the mean vertical velocities (over $5 \mathrm{~mm}^{2}$ ) derived from the PIV data centred at these $x$-locations and at a depth $z=-5.25 \mathrm{~cm}$. It is clear that the low-frequency oscillations, of the order of the forcing frequency $\omega_{f}$, are similar in both datasets, whereas the higher frequencies (i.e. larger than $\omega_{f}$ ) are more pronounced in the ultrasonic probes. This is mainly due to the fact that these high frequencies are localized in the pycnocline itself ( $\omega_{f} \simeq N_{0} / 2$ for EXP22) and have weak signatures below it. Finally, an important feature is the presence of out-of-phase oscillations of the vertical velocity of the pycnocline between its base and its mean position, around $t=170$ or $220 \mathrm{~s}$ in figure $14(b)$ for instance. These are evidence of mode 2 (or higher) internal waves in the pycnocline, in agreement with our findings in $\$ 3.2$.

\section{Appendix B. Modal identification from SAR images}

We present in figure 15 two examples of SAR images with signatures of mode 1 and mode 2 ISWs. The internal waves are moving from right to left. 

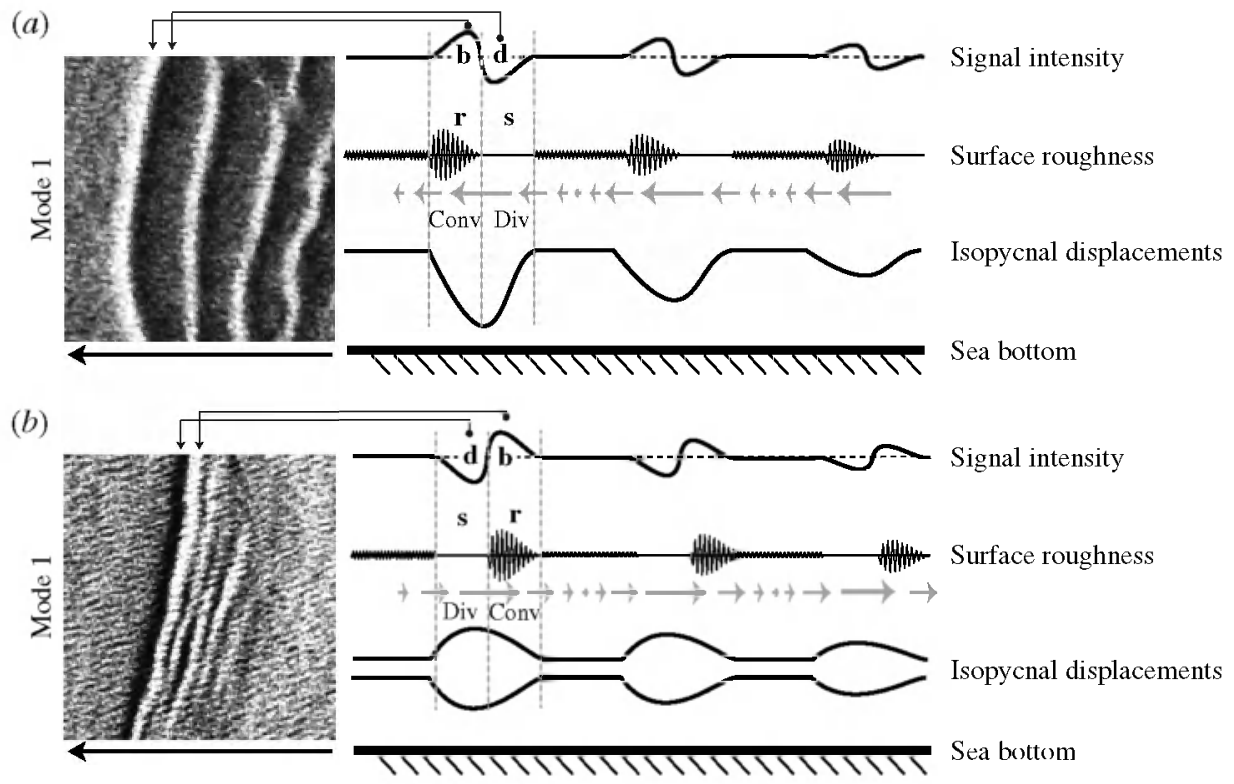

FIGURE 15. Examples of a SAR image with signatures of $(a)$ mode 1 , and $(b)$ mode 2 ISWs.

From top to bottom, the horizontal schematic profiles on the right-hand side of the SAR images represent the following features: the SAR image intensity profile along the ISW propagation direction, with bright enhanced backscatter (b) preceding dark reduced backscatter (d) in the direction of propagation; the surface roughness representation indicating how roughness (r) and smoothness (s) vary along an internal wave train in relation to isopycnal displacements, below; the surface current variability induced by internal waves (note the surface field convergence over the leading slopes of ISWs of depression, and the divergence over the rear slopes); and finally the isopycnal displacements produced by mode 1 or mode 2 internal wave propagation (note the indications of convergence and divergence fields near the surface).

The horizontal profiles represent the same features in both cases. However, one can note how the SAR signatures, roughness field, current field and convergence and divergence patterns are reversed for mode 2 ISWs in relation to mode 1 ISWs, leading to a clear determination of the modal structure of the propagating ISWs.

\section{REFERENCES}

Akylas, T. R., Grimshaw, R. H. J.. Clarke, S. R. \& Tabaei, A. 2007 Reflecting tidal wave beams and local generation of solitary waves in the ocean thermocline. J. Fluid Mech. 593, 297-313.

Azevedo, A., DA Silva, J. C. B. \& New, A. L. 2006 On the generation and propagation of internal solitary waves in the southern Bay of Biscay. Deep-Sea Res. I 53, 927-941.

DA Silva, J. C. B., NEw, A. L. \& AzEvedo, A. 2007 On the role of SAR for observing 'local generation' of internal solitary waves off the Iberian Peninsula. Can. J. Remote Sensing 33 (5). $388-403$.

Da Silva, J. C. B., New, A. L. \& Magalhaes, J. M. 2009 Internal solitary waves in the Mozambique Channel: observations and interpretation. J. Geophys. Res. 114. C05001. 
Da Silva, J. C. B., New, A. L. \& Magalhaes, J. M. 2011 On the structure and propagation of internal solitary waves generated at the Mascarene Plateau in the Indian Ocean. Deep-Sea Res. I 58 (3), 229-240.

Delisi, D. P. \& ORLAnski, I. 1975 On the role of density jumps in the reflexion and breaking of internal gravity beams. J. Fluid Mech. 69, 445-464.

Fincham, A. \& DELERCE, G. 2000 Advanced optimization of correlation imaging velocimetry algorithms. Exp. Fluids 29, S1.

GERKEMA, T. 1996 A unified model for the generation and fission of internal tides in a rotating ocean. J. Mar. Res. 54 (3), 421-450.

GERKEMA, T. 2001 Internal and interfacial tides: beam scattering and local generation of solitary waves. J. Mar. Res. 59 (2), 227-255.

Gostiaux, L. \& Dauxois, T. 2007 Laboratory experiments on the generation of internal tidal beams over steep slopes. Phys. Fluids 19, 028102.

Gostiaux, L., Didelle, H., Mercier, S. \& Dauxois, T. 2007 A novel internal waves generator. Exp. Fluids 42, 123-130.

Grisouard, N., Staquet, C. \& GerkemA, T. 2011 Generation of internal solitary waves in a pycnocline by an internal wave beam: a numerical study. J. Fluid Mech. 676, 491-513.

Horn, D. A., Imberger, J. \& IVEy, G. N. 2001 The degeneration of large-scale interfacial gravity waves in lakes. J. Fluid Mech. 434, 181-207.

JACKSON, C. 2007 Internal wave detection using the moderate resolution imaging spectroradiometer (MODIS). J. Geophys. Res. 112, C11012.

JiANG, C. H. \& MARCUS, P. S. 2009 Selection rules for the nonlinear interaction of internal gravity waves. Phys. Rev. Lett. 102, 124502.

King, B., Zhang, H. P. \& SwINnEY, H. L. 2009 Tidal flow over three-dimensional topography in a stratified fluid. Phys. Fluids 21, 116601.

LeBlond, P. H. \& MYSAK, L. A. 1978 Waves in the Ocean. Elsevier.

MAthur, M. \& PEACock, T. 2009 Internal wave beam propagation in non-uniform stratifications. J. Fluid Mech. 639, 133-152.

Maugé, R. \& Gerkema, T. 2008 Generation of weakly nonlinear nonhydrostatic internal tides over large topography: a multi-modal approach. Nonlinear Process. Geophys. 15, 233-244.

Mercier, M. J., Martinand, D., Mathur, M., Gostiaux, L., Peacock, T. \& Dauxois, T. 2010 New wave generation. $J$. Fluid Mech. 657, 308-334.

Michallet, H. \& BARThéLemy, E. 1997 Ultrasonic probes and data processing to study interfacial solitary waves. Exp. Fluids 22, 380-386.

NEw, A. L. \& DA SILVA, J. C. B. 2002 Remote-sensing evidence for the local generation of internal soliton packets in the central Bay of Biscay. Deep-Sea Res. 49 (5), 915-934.

New, A. L. \& Pingree, R. D. 1990 Large-amplitude internal soliton packets in the central Bay of Biscay. Deep-Sea Res. 37, 513-524.

New, A. L. \& PIngReE, R. D. 1992 Local generation of internal soliton packets in the central Bay of Biscay. Deep-Sea Res. 39, 1521-1534.

Tabaei, A. \& Akylas, T. R. 2003 Nonlinear internal gravity wave beams. J. Fluid Mech. 482, 141-161.

Tabaei, A., Akylas, T. R. \& Lamb, K. G. 2005 Nonlinear effects in reflecting and colliding internal wave beams. J. Fluid Mech. 526, 217-243.

Thomas, N. H. \& Stevenson, T. N. 1972 A similarity solution for viscous internal waves. J. Fluid Mech. 54, 495-506.

Thorpe, S. A. 1998 Nonlinear reflection of internal waves at a density discontinuity at the base of the mixed layer. J. Phys. Oceanogr. 28, 1853-1860. 